\title{
O problema do potencial eletrostático para condições de contorno de uma caixa como ferramenta de aprendizado de Eletromagnetismo em um curso de graduação em Física
}

The electrostatic potential problem for boundary conditions of a box as a tool for learning electromagnetism in an undergraduate physics course

\author{
Deyvid W. da M. Pastana ${ }^{1}$, Vinicius B. Neves ${ }^{1}$, Manuel E. Rodrigues ${ }^{* 1,2} @$ \\ ${ }^{1}$ Universidade Federal do Pará, Faculdade de Ciências Exatas e Tecnologia, Abaetetuba, PA, Brasil. \\ ${ }^{2}$ Universidade Federal do Pará, Faculdade de Física, Programa de Pós-Graduação em Física, Belém, PA, Brasil.
}

Recebido em 23 de fevereiro de 2021. Revisado em 08 de julho de 2021. Aceito em 12 de julho de 2021.

\begin{abstract}
Desde que James Clerck Maxwell publicou seu brilhante trabalho unificando a eletricidade e magnetismo surgiram, a partir de então, inúmeras aplicações práticas do mesmo. Este artigo discute o potencial elétrico bidimensional e tridimensional, obtidos a partir da solução da equação de Laplace, assim como a solução para os campos elétrico e magnético para a equação de Helmholtz com condições iniciais específicas. Explicitamos a utilização do teorema de superposição em um exemplo claro de eletrostática. Em particular, foi estudado o problema do potencial elétrico em uma caixa retangular com condições de contorno de Dirichlet, fazendo uma aplicação em guias de onda e cavidades ressonantes. O software Mathematica foi usado para obter os gráficos do potencial e campo elétrico.
\end{abstract}

Palavras chave: equação de Laplace, potencial eletrostático, condições de contorno retangulares.

Ever since James Clerck Maxwell published his brilliant work unifying electricity and magnetism, numerous practical applications of it have arisen. This paper discusses the two- and three-dimensional electric potentials obtained from the solution of Laplace's equation, as well as the solution to the electric and magnetic fields for the Helmholtz equation with specific initial conditions. We explain the use of the superposition theorem in a clear electrostatics example. In particular, the problem of the electric potential in a rectangular box with Dirichlet boundary conditions was studied, making an application to waveguides and resonant cavities. Mathematica software was used to obtain the plots of the potential and electric field.

Keywords: Laplace equation, electrostatic potential, rectangular boundary conditions.

\section{Introdução}

O aprendizado de ferramentas matemáticas no ensino médio é de suma importância para qualquer aluno. Quando esse aluno ingressa no nível superior, em um curso de Licenciatura ou Bacharelado em Física, ele nessecita lidar com uma das mais potentes ferramentas nas ciências, o cálculo diferencial e integral. Logo cedo no curso esse aluno se depara com uma das leis mais conhecidas na Física Clássica, a segunda lei de Newton, a aqual é definida através de uma equação diferencial ordinária de segunda ordem. Assim como no início, todo decorrer do curso de Física utiliza essa mesma linguagem, exigindo uma crescente prática e aprendizado dessa ferramente pelo aluno.

A generalização de equações diferenciais ordinárias, são as equações diferenciais parciais. Uma das primeiras que um aluno do cruso de Física se depara é a equação de Laplace, uma equação diferencial parcial linear de segunda ordem. Seja no estudo do calor, ou em uma

\footnotetext{
* Endereço de correspondência: esialg@gmail.com
}

disciplina de Métodos Matemáticos para Física, o aluno começa a resolver essa equação. Especificamente, em uma disciplina de Eletromagnetismo Clássico, um aluno do curso de Física deve logo resolver essa equação, e também interpretá-la fisicamente para determinadas condições iniciais, como para uma placa bidimensional infinita, ou finita, para uma caixa retangular, etc. Nesse caso, o aluno necessita de conhecimento em equações diferenciais parciais e série de Fourier. Essas técnicas iniciais servem como base na eletrodinâmica e em várias outras áreas da Física.

O objetivo principal desse artigo é estudar as soluções da equação de Laplace em duas e três dimensões, com condições iniciais em uma placa retangular finita e uma caixa retangular. A resolução do problema da caixa retangular é um pré-requisito para a resolução do problema dinâmico de uma onda se propagando em uma caixa retangular condutora, o qual é entendido resolvendo-se a equação de Helmholtz, a qual pode ser considerada como uma generalização da equação de Laplace para o caso de campos dinâmicos. O método de separação de variáveis utilizado aqui é idêntico ao 
adotado nos livros textos mais conhecidos de Eletromagnetismo [3 6], com a diferença que vamos explicitar a utilização do princípio de superposição, o qual estabelece que a combinação linear de duas ou mais soluções da equação de Laplace, também é uma solução da mesma. Usaremos esse princípio de equações diferenciais parciais na resolução de um problema um pouco mais elaborado que os exemplos vistos nos livros textos de cursos de Eletromagnetismo Clássico. Podemos pensar primeiro no seguinte exemplo, uma caixa retangular condutora em que somente uma das seis paredes está sujeita a um potencial eletrostático $V_{1}$, as outras estão neutras. Queremos então encontrar o potencial dentro dessa caixa. Esse é um típico problema eletrostático, onde devemos resolver a equação de Laplace para o potencial que satisfaz uma condição de contorno, ou fronteira, específica, a qual é, nesse caso, o potencial eletrostático é sempre zero em todas as cinco paredes da caixa, e é $V_{1}$ em apenas uma das paredes. Esse problema é muito interessante, o qual pode ser comparado ao caso do potencial dentro de um forno micro-ondas, e encontra-se resolvido na seguinte referência [6] (em [3] encontramos resolvido o problema de um tubo retangular infinitamente longo). Agora podemos ir um pouco mais longe. Se tivermos agora uma caixa condutora retangular em que duas das faces (chamaremos 1 e 2) estejam sujeitas a potenciais eletrostáticos, uma sujeita a $V_{1}$ e a segunda a $V_{2}$, e todas as outras quatro restantes estão neutras. Podemos resolver esse novo problema mais facilmente usando o princípio da superposição. Primeiro resolvemos a equação de Laplace para um potencial $V_{1}^{(1)}$ que possui a seguinte condição de contorno: cinco faces da caixa retangular estão neutras e somente a face 1 está sujeita ao potencial eletrostático $V_{1}$. Essa é a primeira solução. Depois resolvemos a equação de Laplace para um novo potencial $V_{2}^{(2)}$, o qual possui a condição de contorno que somente a face 2 está sujeita ao potencial eletrostático $V_{2}$, e todas as outras estão neutras. Por fim, usando o princípio de superposição, encontramos que a solução, que tem a condição de contorno em que as faces 1 , sujeita a $V_{1}$, e 2 , sujeita a $V_{2}$, é a combinação linear $a V_{1}^{(1)}+$ $b V_{2}^{(2)}$. O que nos propomos a fazer, aquilo que não se encontra nos livros textos de Eletromagnetismo Clássico, como [3 7], é resolver um exercício muito ilustrativo, retirado do capítulo 6 de [6], exercício 6.2, que fornece o problema de uma caixa condutora retangular em que a face 1 está sujeita a um potencial eletrostático $V_{1}$, a face 2 a $V_{2}$, e por fim a face 3 ao potencial $V_{3}$, enquanto que as restantes estão neutras (para que o leitor tenha uma melhor compreensão do problema proposto, ver a seção 4, onde o problema está explicitado). Resolver esse exercício é uma excelente oportunidade de aprendizado da utilização clara do princípio da superposição. Faremos isso mais a frente. Para poder resolver esse problema tridimensional, é recomendável que, para fins didáticos, iniciememos resolvendo um problema bidimensional, de um tubo retangular infinito, em que dois lados estão sujeitos a um potencial eletrostático $V_{0}$, e os outros estão aterrados. Esse problema está resolvido como exemplo na referência [3, exemplo 3.4. Então, no caso bidimensional, nós fazemos apenas uma revisão como preparação para o problema tridimensional.

O problema do potencial eletrostático é na verdade um caso particular de um problema mais geral conhecido como problema de Dirichlet para uma equação diferencial parcial elíptica de segunda ordem, que pode ser formulado da seguinte forma (corolário 6.17 da página $145 \mathrm{em}$ [13])

$$
\begin{aligned}
& \sum_{i, j=1}^{N} a_{i j}(X) \frac{\partial^{2} u}{\partial X_{i} \partial X_{j}}+\Sigma_{i=1}^{N} b_{i}(X) \frac{\partial u}{\partial X_{i}}+ \\
& \quad+c(X) u=f(X) \text { em } \Omega, \\
& u(X)=g(X) \text { sobre } \partial \Omega,
\end{aligned}
$$

onde $X$ são as variáveis independentes, $\Omega$ um aberto limitado do $\mathbb{R}^{N}, u(X)$ um potencial em que $u \in C^{2}(\Omega)$ (uma função contíniua em que a primeira e segunda derivadas também o são) e também $f \in C^{2}(\Omega)$. O caso geral de equação elíptica recai em diversos outros casos particulares muito conhecidos.

Tomando $X=\{x, y, z, t\}, a_{i j}=\delta_{i j}\left(\delta_{i j}\right.$ sendo a delta de Kronecker, vale 1 para os índices $i=j$ e zero para $i \neq j$, e $i, j=1,2,3)$ só com componentes diagonais espaciais, $b_{i}$ só com a componente $b_{t}=-1$ (as outras componentes sendo zero) e $c=f=0$, temos a equação de difusão

$$
\begin{array}{r}
\Delta u=\partial_{t} u \text { em } \Omega, \\
u(X)=g(X) \text { sobre } \partial \Omega,
\end{array}
$$

onde $\Delta$ é o operador laplaciano $\Delta=\partial_{x}^{2}+\partial_{y}^{2}+\partial_{z}^{2}$. Essa equação tem inúmeras aplicações, inclusive na condução de calor [1].

Tomando $X=\{x, y, z, t\}, a_{i j}=\delta_{i j}$ só com componentes diagonais espaciais e $a_{t t}=-1 / v^{2}, b_{i}=f=0$ e $c=m^{2}$, temos a equação de Klein-Gordon

$$
\begin{array}{r}
\Delta u-\frac{1}{v^{2}} \partial_{t}^{2} u+m^{2} u=0 \text { em } \Omega, \\
u(X)=g(X) \text { sobre } \partial \Omega .
\end{array}
$$

Essa equação é largamente utilizada em Teoria Quântica de Campos [7]. Essa equação recai na famosa equação de onda para $m^{2}=0$. Em particular, tomando o problema geral de Dirichlet com $m^{2}=0$ em (5), na eletrodinâmica, considere um domínio $\Omega$ correspondente à uma caixa retangular e $g\left(\mathbb{R}^{3}\right)$ representar suas condições de contorno nessa superfície. Seja $u_{i}=\mathcal{E}_{i}(\vec{r}, t)$, obtemos a equação de Helmholtz para propagação de onda no interior de uma caixa retangular, cuja solução é da forma $\mathcal{E}_{i}(\vec{r}, t)=E_{i}(\vec{r}) e^{-i \omega t}$. Vale o mesmo para o campo magnético. Como vimos acima, esse é um problema que pode recair na equação de Laplace quando fazemos $k=0, \operatorname{logo} \omega=0$, e é equivalente a fazer $t=0$ na solução. Se considerarmos que a superfície da caixa 
é constituída de um material bom condutor, a onda que propagando-se no interior da caixa deve satisfazer a condição de campo nulo, as componentes do campo devem se anular nas paredes perpendiculares a sua direção (isso é idêntico ao problema de um campo confinado a uma certa região no efeito Casimir dinâmico [9]). Portanto, se $\vec{E}(\vec{r})$ satisfazer a condição de campo nulo, pode ser utilizado na solução da equação de Helmholtz, de forma que o campo eletrostático possa ser retomado em valores próximos do tempo inicial. Portanto, a equação de Laplace tem aplicação direta em guias de ondas e cavidades ressonantes. Alguns métodos mais gerais e problemas mais amplos podem ser consultados em [8]. O interessante é que esse mesmo problema é corriqueiro em Termodinâmica, Gravitação, Hidrodinâmica, e muitas outras áreas da Física.

Um outro caso particular da equação elíptica (1) bastante conhecido em eletrostática é quando tomamos $a_{i j}$ só com componentes diagonais espaciais, $b_{i}=c=0$, e $f=-\rho / \epsilon, u=V(x, y, z)$, então temos

$$
\begin{aligned}
& \frac{\partial^{2} V}{\partial x^{2}}+\frac{\partial^{2} V}{\partial y^{2}}+\frac{\partial^{2} V}{\partial z^{2}}=-\frac{\rho(\vec{r})}{\epsilon} \text { em } \Omega \\
& V(x, y, z)=g(x, y, z) \text { sobre } \partial \Omega .
\end{aligned}
$$

Essa é a equação de Poisson da eletrostática. Quando tomamos a densidade de cargas igual a zero, temos finalmente a equação de Laplace

$$
\begin{aligned}
& \frac{\partial^{2} V}{\partial x^{2}}+\frac{\partial^{2} V}{\partial y^{2}}+\frac{\partial^{2} V}{\partial z^{2}}=0 \text { em } \Omega \\
& V(x, y, z)=g(x, y, z) \text { sobre } \partial \Omega
\end{aligned}
$$

Trataremos nesse artigo unicamente das equações de Laplace (9) e de Helmholtz.

Esse artigo segue os métodos descritos nas seguintes referências [3 4], 10]. A abordagem será feita da seguinte forma: na seção 2 as equação de Laplace tridimensional é presentada e resolvida seguindo os passos de [6]; em 3 o problema do potencial bidimensional é resolvido fazendo uso do coeficiente de Fourier [11]; a seção 4 aborda um problema tridimensional, o qual é solucionado com o coeficiente de Fourier em duas dimensões, e aplicando o princípio de superposição para EDPs homogêneas [12]; por fim, na seção 5 é mostrado como o campo eletrostático advindo da equação de Laplace pode ser aplicado em propagação de ondas eletromagnéticas.

\section{Equação de Laplace}

Em Eletrostática é muito importante poder calcular o campo elétrico de uma certa distribuição de cargas, em uma certa região do espaço. Porém o campo elétrico é uma grandeza vetorial que está refém de uma soma vetorial, que é, em geral, mais complicada que uma soma escalar. Portanto, se pudermos relacionar uma grandeza escalar ao campo elétrico, poderemos efetuar os cálculos através dessas funções escalares e ao final do problema retornar a grandeza de interesse, o campo elétrico, de forma que o procedimento fica bastante facilitado. Indubitavelmente, essa associação é possível e nos leva diretamente a equação de Laplace. Nessa seção vamos seguir os passos básicos para obter a equação de Laplace, considerando a definição de força, campo e potencial elétrico.

Nós vamos utilizar basicamente a referência [6] para estabelecer a equação de Laplace e resolvê-la em geral, sem condições de contorno especificadas.

A força eletrostática $\vec{F}_{e}$ exercida sobre a carga $Q$, situada em $\vec{r}$, pela carga $q$, situada em $\vec{r}^{\prime}$, no vácuo, é dada pela lei de Coulomb [3, 5, 7]

$$
\vec{F}_{e}(\vec{r})=\frac{q Q}{4 \pi \varepsilon_{0}} \frac{\left(\vec{r}-\vec{r}^{\prime}\right)}{\left|\vec{r}-\vec{r}^{\prime}\right|^{3}},
$$

onde $\left|\vec{r}-\vec{r}^{\prime}\right|$ é o módulo da distância definida pelos pontos $\vec{r}$ e $\vec{r}^{\prime}$. É evidente que se as cargas têm sinais opostos a força definida acima será atrativa, ao passo que, se tiverem o mesmo sinal, a força será repulsiva, como tem que ser.

Quando não tivermos apenas duas cargas interagindo, e sim várias cargas pontuais $q_{i}$, nas posições $\vec{r}_{i}$, a lei de Coulomb torna-se a seguinte soma vetorial

$$
\vec{F}_{e}=\frac{Q}{4 \pi \varepsilon_{0}} \sum_{i=1}^{n} \frac{q_{i}\left(\vec{r}-\vec{r}_{i}\right)}{\left|\vec{r}-\vec{r}_{i}\right|^{3}}
$$

e se tivermos uma distribuição de cargas $q^{\prime}$ em um certo volume $V^{\prime}$, com uma densidade $\rho\left(\vec{r}^{\prime}\right)$, a força é escrita como

$$
\vec{F}_{e}=\frac{Q}{4 \pi \varepsilon_{0}} \int_{V^{\prime}} \rho\left(\vec{r}^{\prime}\right) \frac{\left(\vec{r}-\vec{r}^{\prime}\right)}{\left|\vec{r}-\vec{r}^{\prime}\right|^{3}} d V^{\prime}
$$

e a integral se estende sobre todo o volume $V^{\prime}$. Esta expressão depende da carga de prova $Q$. Outro sim, seria melhor utilizar apenas o efeito da distribuição de cargas, e, assim, definimos o campo elétrico $\vec{E}$ como

$$
\vec{E}=\frac{\vec{F}_{e}}{Q}
$$

que não depende da carga de prova. Com isso em mãos, o campo gerado por uma distribuição de cargas em um ponto $\vec{r}$ do espaço é

$$
\vec{E}=\frac{1}{4 \pi \varepsilon_{0}} \int_{V^{\prime}} \rho\left(\vec{r}^{\prime}\right) \frac{\left(\vec{r}-\vec{r}^{\prime}\right)}{\left|\vec{r}-\vec{r}^{\prime}\right|^{3}} d V^{\prime}
$$

A expressão (11) nos dá o campo elétrico para qualquer distribuição de cargas e é a partir dela que obteremos o potencial elétrico.

O operador diferencial $\nabla$ em coordenadas retangulares é dado por

$$
\nabla=\hat{i} \frac{\partial}{\partial x}+\hat{j} \frac{\partial}{\partial y}+\hat{k} \frac{\partial}{\partial z}
$$


Quando esse operador age sobre uma função escalar $\phi$, o resultado é uma função vetorial associada à $\phi$. Tomamos portanto, o gradiente da função

$$
\phi(\vec{r})=\frac{1}{\left|\vec{r}-\vec{r}^{\prime}\right|},
$$

ou seja, iremos calcular

$$
\nabla\left[\frac{1}{\left|\vec{r}-\vec{r}^{\prime}\right|}\right]
$$

o que dá

$$
\nabla\left[\frac{1}{\left|\vec{r}-\vec{r}^{\prime}\right|}\right]=-\frac{\left(\vec{r}-\vec{r}^{\prime}\right)}{\left|\vec{r}-\vec{r}^{\prime}\right|^{3}} .
$$

Agora, usando a equação 12 na 11 , obtemos

$$
\vec{E}(\vec{r})=-\frac{1}{4 \pi \varepsilon_{0}} \int_{V^{\prime}} \rho\left(\vec{r}^{\prime}\right) \nabla\left[\frac{1}{\left|\vec{r}-\vec{r}^{\prime}\right|}\right] d V^{\prime},
$$

e como a densidade de cargas é restritamente função de $\vec{r}^{\prime}$, podemos tirar o operador para fora da integral, justamente porque ele não age sobre $\rho\left(\vec{r}^{\prime}\right)$. Ou seja,

$$
\vec{E}(\vec{r})=-\nabla\left\{\frac{1}{4 \pi \varepsilon_{0}} \int_{V^{\prime}}\left[\frac{\rho\left(\vec{r}^{\prime}\right)}{\left|\vec{r}-\vec{r}^{\prime}\right|}\right] d V^{\prime}\right\},
$$

e a expressão entre chaves é uma grandeza escalar, que é definida como o potencial elétrico $V(\vec{r})$ da distribuição de cargas $\rho\left(\vec{r}^{\prime}\right)$, isto é,

$$
V(\vec{r})=\frac{1}{4 \pi \varepsilon_{0}} \int_{V^{\prime}}\left[\frac{\rho\left(\vec{r}^{\prime}\right)}{\left|\vec{r}-\vec{r}^{\prime}\right|}\right] d V^{\prime},
$$

e assim, potencial e campo elétrico estão conectados pela relação

$$
\vec{E}=-\nabla V
$$

Portanto, quando calculamos o potencial elétrico, podemos obter o campo, tomando o gradiente desse potencial com sinal trocado.

Para dar prosseguimento rumo a equação de Laplace, precisamos do teorema do divergente [3, 5, 6]

$$
\int_{V} \nabla \cdot \vec{A} d V=\oint_{S} \vec{A} \cdot \hat{n} d S
$$

Esta propriedade vale para qualquer função vetorial inclusive o campo elétrico $\vec{E}$. Vamos usar a equação 15 para essa grandeza. Portanto, temos

$$
\int_{V} \nabla \cdot \vec{E} d V=\oint_{S} \vec{E} \cdot \hat{n} d S
$$

Para encontrar $\nabla \cdot \vec{E}$, usamos a equação 11

$$
\nabla \cdot \vec{E}=\nabla \cdot\left\{\frac{1}{4 \pi \varepsilon_{0}} \int_{V^{\prime}} \rho\left(\vec{r}^{\prime}\right) \frac{\left(\vec{r}-\vec{r}^{\prime}\right)}{\left|\vec{r}-\vec{r}^{\prime}\right|^{3}} d V^{\prime}\right\},
$$

e como o operador $\nabla$ não age sobre a densidade de cargas, a equação 16 se torna

$$
\nabla \cdot \vec{E}=\frac{1}{4 \pi \varepsilon_{0}} \int_{V^{\prime}} \rho\left(\vec{r}^{\prime}\right) \nabla \cdot \frac{\left(\vec{r}-\vec{r}^{\prime}\right)}{\left|\vec{r}-\vec{r}^{\prime}\right|^{3}} d V^{\prime}
$$

o que resulta

$$
\nabla \cdot \frac{\left(\vec{r}-\vec{r}^{\prime}\right)}{\left|\vec{r}-\vec{r}^{\prime}\right|^{3}}=4 \pi \delta\left(\vec{r}-\vec{r}^{\prime}\right) .
$$

Tendo a equação 18 , voltamos a expressão 16 , para encontrar

$$
\nabla \cdot \vec{E}=\frac{1}{4 \pi \varepsilon_{0}} \int_{V^{\prime}} \rho\left(\vec{r}^{\prime}\right) 4 \pi \delta\left(\vec{r}-\vec{r}^{\prime}\right) d V^{\prime}=\frac{1}{\varepsilon_{0}} \rho(\vec{r}),
$$

e, então, obtemos a equação 19

$$
\nabla \cdot \vec{E}=\frac{\rho}{\varepsilon_{0}}
$$

que é uma das quatro equações de Maxwell, na forma diferencial.

Como já vimos, campo e potencial elétrico estão relacionados pela equação (14). Substituindo essa equação na expressão (19, temos a equação de Poisson [20] [3, 5, 7]

$$
\nabla^{2} V=-\frac{\rho}{\varepsilon_{0}}
$$

onde $\nabla^{2}$ é o operador Laplaciano. A equação de Poisson nos fornece o potencial onde há uma distribuição $\rho$ de cargas.

Finalmente, se na região onde será calculado o potencial estiver livre de cargas, esta equação se torna

$$
\nabla^{2} V=0
$$

que é a equação de Laplace para o potencial elétrico [3, 5,7]. Note que a região está livre de cargas, no entanto ela está submetida ao potencial gerado por cargas situadas em outra região. Apesar de parecer simples, esta equação é demasiadamente importante e muito rica fisicamente.

\subsection{Propriedades gerais das Equação de Laplace}

Antes de prosseguirmos, mostraremos algumas propriedades gerais da equação (21), a da superposição de soluções e da unicidade das soluções. Essas propriedades estão relacionadas com os seguintes teoremas:

Teorema I [6, 12] Se $V_{1}, V_{2}, \cdots, V_{n}$ forem todos soluções da equação de Laplace, então

$$
V=C_{1} V_{1}+C_{2} V_{2}+\cdots+C_{n} V_{n}
$$

onde os $C_{i}$ são constantes arbitrárias, será também uma solução.

Teorema II 6 Duas soluções da equação de Laplace que satisfazem as mesmas condições de contorno diferem, no máximo, por uma constante aditiva. 
Através do teorema I podemos superpor várias soluções da equação de Laplace, de forma que a solução resultante satisfaça um dado conjunto de condições de contorno. Esse vai ser nosso principal tema, exemplificando uma utilização desse teorema para obter uma solução geral de um caso de equação de Laplace.

\subsection{Equação de Laplace em coordenadas retangulares tridimensionais}

Em três dimensões a equação 21] é escrita como

$$
\nabla^{2} V=\frac{\partial^{2} V}{\partial x}+\frac{\partial^{2} V}{\partial y}+\frac{\partial^{2} V}{\partial z}=0
$$

Para resolvermos essa equação diferencial parcial, usaremos o método de separação de variáveis [6, 12]. Supondo uma solução do tipo

$$
V=X(x) Y(y) Z(z)
$$

adquirimos

$$
Y Z \frac{d^{2} X}{d x^{2}}+X Z \frac{d^{2} Y}{d y^{2}}+X Y \frac{d^{2} Z}{d z^{2}}
$$

dividindo tudo por $X(x) Y(y) Z(z)$ e isolando a parte em $Z$ fica

$$
\frac{1}{X} \frac{d^{2} X}{d x^{2}}+\frac{1}{Y} \frac{d^{2} Y}{d y^{2}}=-\frac{1}{Z} \frac{d^{2} Z}{d z^{2}}
$$

O lado esquerdo depende apenas de $x$ e $y$ e o lado direito apenas de $Z$. Para que sejam iguais é preciso que ambos sejam uma constante, que convencionamos como $-k^{2}$. Em vista disso, temos duas equações

$$
\frac{1}{X} \frac{d^{2} X}{d x^{2}}+\frac{1}{Y} \frac{d^{2} Y}{d y^{2}}=-k^{2}
$$

e

$$
-\frac{1}{Z} \frac{d^{2} Z}{d z^{2}}=-k^{2}
$$

Resolvendo a equação 23 primeiro, temos

$$
\frac{d^{2} Z}{d z^{2}}-k^{2} Z=0
$$

em que a solução geral dessa equação diferencial para $k \neq 0$ é

$$
Z_{k \neq 0}(z)=f e^{k z}+g e^{-k z}
$$

Para $k=0$ a solução da equação 24 é

$$
Z_{k=0}(z)=f_{0}+g_{0} z
$$

Para resolver a equação 22 , isolamos o termo em $X$, resultando

$$
\frac{1}{X} \frac{d^{2} X}{d x^{2}}=-k^{2}-\frac{1}{Y} \frac{d^{2} Y}{d y^{2}}
$$

Esta equação também está separada e deve ser igual a uma constante, que chamaremos de $-l^{2}$. Então, temos novamente duas equações, ou seja,

$$
\frac{1}{X} \frac{d^{2} X}{d x^{2}}=-l^{2}
$$

e

$$
-k^{2}-\frac{1}{Y} \frac{d^{2} Y}{d y^{2}}=-l^{2}
$$

Resolvendo a equação (27) para $X$, obtemos a solução

$$
X_{l \neq 0}(x)=a \cos (l x)+b \sin (l x)
$$

Para $l=0$, a solução é

$$
X_{l=0}(x)=a_{0}+b_{0} x
$$

A equação diferencial (28), pode ser reescrita como

$$
\frac{d^{2} Y}{d y^{2}}+\left(k^{2}-l^{2}\right) Y=0
$$

Definindo

$$
\lambda^{2}=k^{2}-l^{2}
$$

e a equação diferencial se torna

$$
\frac{d^{2} Y}{d y^{2}}+\lambda^{2} Y=0
$$

que tem como solução, quando $\lambda \neq 0$ a equação

$$
Y_{\lambda \neq 0}(y)=c \cos (\lambda y)+d \sin (\lambda y)
$$

Quando $\lambda=0$ a equação 32 se torna

$$
\frac{d^{2} Y}{d y^{2}}=0
$$

com a solução

$$
Y_{\lambda=0}(y)=c_{0}+d_{0} y
$$

A solução geral é formada pela combinação das equações para $X, Y$ e $Z$ obtidas acima. Quando $k=l=$ $\lambda=0$, a solução é

$$
\left.V_{0,0}=\left[a_{0,0}+b_{0,0} x\right]\left[c_{0,0}+d_{0,0} y\right]\left[f_{0,0}+g_{0,0} z\right]\right]
$$

Quando $l=0$ e $k \neq 0$, por 31 , acontece que $\lambda^{2}=k^{2}$, de modo que $\lambda= \pm k$. Portanto a solução é

$$
\begin{aligned}
V_{k, 0}= & {\left[a_{k, 0}+b_{k, 0} x\right]\left[c_{k, 0} \cos (k y)+d_{k, 0} \sin (k y)\right] } \\
& \times\left[f_{k, 0} e^{k z}+g_{k, 0} e^{-k z}\right]
\end{aligned}
$$

Agora se $k=0$ e $l \neq 0$, pela equação (31), ocorre $\lambda^{2}=-l^{2}$, de maneira que $\lambda= \pm i l$. Assim, a solução para o potencial é

$$
\begin{aligned}
V_{0, l}= & {\left[a_{0, l} \cos (l x)+b_{0, l} \sin (l x)\right]\left[c_{0, l} e^{l y}+d_{0,0} e^{-l y}\right] } \\
& \times\left[f_{0, l}+g_{0, l} z\right]
\end{aligned}
$$


Quando $k=l \neq 0, \lambda=0$, a solução é dada por

$$
\begin{aligned}
V_{k, k}= & {\left[a_{k, k} \cos (k x)+b_{k, k} \sin (k x)\right] } \\
& \times\left[c_{k, k}+d_{k, k} y\right]\left[f_{k, k} e^{k z}+g_{k, k} e^{-k z}\right]
\end{aligned}
$$

E a ultima solução, para quando $k \neq l, k \neq 0$ e $l \neq 0$ é dada por

$$
\begin{aligned}
V_{k, l}= & {\left[a_{k, l} \cos (l x)+b_{k, l} \sin (l x)\right]\left[c_{k, l} \cos (\lambda y)\right.} \\
& \left.+d_{k, l} \sin (\lambda y)\right] \times\left[f_{k, l} e^{k z}+g_{k, l} e^{-k z}\right]
\end{aligned}
$$

lembrando que, por (31), $\lambda^{2}=k^{2}-l^{2}$. A solução geral é a soma de (35), 36), (37), 38) e (39). Ou seja,

$$
V(x, y, z)=V_{0,0}+V_{k, 0}+V_{0, l}+V_{k, k}+V_{k, l}
$$

As constantes que surgiram durante a obtenção das soluções, dependem de condições de contorno de cada problema específico.

\subsection{Coeficiente da série de Fourier}

Nos problemas de equação de Laplace para uma determinada condição de contorno, é comum que em algum momento uma condição de contorno resulte em uma constante múltipla da função seno ou cosseno, cujo argumento pode ser proporcional a um número inteiro e a pi. Nesses casos, dada a periodicidade dessas funções, a solução é dada em termos de uma série de Fourier. Portanto, o coeficiente da série de Fourier é uma ferramenta útil na solução de problemas de potenciais eletrotáticos com condições de contorno. Vamos determinar o coeficiente de Fourier para uma função bidimensional nessa subseção.

Seja definida a série dupla de Fourier em senos como

$$
f(x, y)=\sum_{m=1}^{\infty} \sum_{n=1}^{\infty} A_{m, n} \sin (m x) \sin (n y)
$$

Para encontrarmos o coeficiente $A_{m, n}$, multiplicamos os dois membros da equação 41 por $\sin (p x) \sin (q y)$. $\mathrm{Ou}$ seja,

$$
\begin{aligned}
f(x, y) & \sin (p x) \sin (q y) \\
= & \sum_{m=1}^{\infty} \sum_{n=1}^{\infty} A_{m, n} \\
& \times[\sin (m x) \sin (n y) \sin (p x) \sin (q y)]
\end{aligned}
$$

Agora, integrando nos intervalos $0 \leq x \leq \pi$ e $0 \leq y \leq$ $\pi$, também em ambos os membros, temos

$$
\begin{aligned}
\int_{0}^{\pi} \int_{0}^{\pi} f(x, y) \sin (p x) \sin (q y) \\
=\sum_{m=1}^{\infty} \sum_{n=1}^{\infty} A_{m, n} \\
\quad \times \int_{0}^{\pi} \sin (m x) \sin (p x) d x \int_{0}^{\pi} \sin (n y) \sin (q y) d y
\end{aligned}
$$

A integral em $x$, fica

$$
\begin{aligned}
\int_{0}^{\pi} \sin (m x) \sin (p x) d x= & -\frac{1}{2} \int_{0}^{\pi} \cos [(m+p) x] d x \\
& +\frac{1}{2} \int_{0}^{\pi} \cos [(m-p) x] d x
\end{aligned}
$$

Na primeira integral em 443 , temos

$$
\frac{1}{2} \int_{0}^{\pi} \cos [(m+p) x]=0
$$

para todo $m, p \in \mathbb{Z}$.

Na segunda integral em (43), obtemos

$$
\frac{1}{2} \int_{0}^{\pi} \cos [(m-p) x] d x=0
$$

quando $m \neq p$. Para $m=p$, temos

$$
\frac{1}{2} \int_{0}^{\pi} \cos [(m-p) x] d x=\frac{1}{2} \int_{0}^{\pi} d x=\frac{\pi}{2}
$$

E assim, apenas quando $m=p$ temos um termo nãonulo. Para representar isso, podemos utilizar o símbolo chamado Delta de Kronecker, definido por

$$
\delta_{i, j}= \begin{cases}1, & \text { se } i=j \\ 0, & \text { se } i \neq j\end{cases}
$$

Com isso, a integral em 43 fica

$$
\int_{0}^{\pi} \sin (m x) \sin (p x) d x=\frac{\pi}{2} \delta_{m, p}
$$

Analogamente, temos o resultado da segunda integral em 42

$$
\int_{0}^{\pi} \sin (n y) \sin (q y) d y=\frac{\pi}{2} \delta_{n, q}
$$

Portanto, juntando 47 e 48 em 42 obtemos

$$
\begin{aligned}
& \int_{0}^{\pi} \int_{0}^{\pi} f(x, y) \sin (p x) \sin (q y) \\
& \quad=\frac{\pi^{2}}{4} \sum_{m=1}^{\infty} \sum_{n=1}^{\infty} A_{m, n} \delta_{m, p} \delta_{n, q}
\end{aligned}
$$

onde

$$
\sum_{m=1}^{\infty} \sum_{n=1}^{\infty} A_{m, n} \delta_{m, p} \delta_{n, q}=A_{p, q}
$$

portanto,

$$
A_{p, q}=\frac{4}{\pi^{2}} \int_{0}^{\pi} \int_{0}^{\pi} f(x, y) \sin (p x) \sin (q y) d x d y .
$$




\section{Revisão do Problema Bidimensional do Potencial Eletrostático}

Nesta seção iremos tratar de um problema tridimensional que, por simetria, recai em um problema matemático bidimensional. Esse problema é resolvido como um exemplo 3.4 do livro [3], sendo que aqui usamos essa revisão como uma etapa para o aprendizado sobre equação de Laplace.

Considere um tubo retangular oco infinito na direção $z$, conforme a Figura 1. O tubo é constituído de duas placas infinitas paralelas ao plano $x z$, as quais são aterradas. Essas placas estão nos planos $y=0$ e $y=a$. Agora, considera mais duas placas infinitas paralelas ao plano $y z$, situadas em $x=-b$ e $x=b$. Essas últimas placas estão isoladas das outras duas através de uma fina camada isolante colocada em cada canto do tubo, e estão sujeitas a um potencial $V_{0}$ constante. A questão é: qual o potencial eletrostático dentro do tubo?

Como a configuração das placas resulta em um problema que não depende da coordenada $z$, esse problema pode ser representado matematicamente pelas seguintes condições de contorno

$$
\begin{cases}V(x, 0)=0 & V(x, a)=0 \\ V(-b, y)=V_{0} & V(b, y)=V_{0}\end{cases}
$$

O problema então é solucionado resolvendo-se a equação de Laplace em duas dimensões.

Portanto, trata-se de um potencial $V(x, y)=$ $X(x) Y(y)$ sujeito a equação de Laplace em duas dimensões,

$$
Y \frac{d^{2} X}{d x^{2}}+X \frac{d^{2} Y}{d y^{2}}=0
$$

Multiplicando os membros da equação 51 por $1 / X Y$, obtemos

$$
\frac{1}{X} \frac{d^{2} X}{d x^{2}}+\frac{1}{Y} \frac{d^{2} Y}{d y^{2}}=0
$$

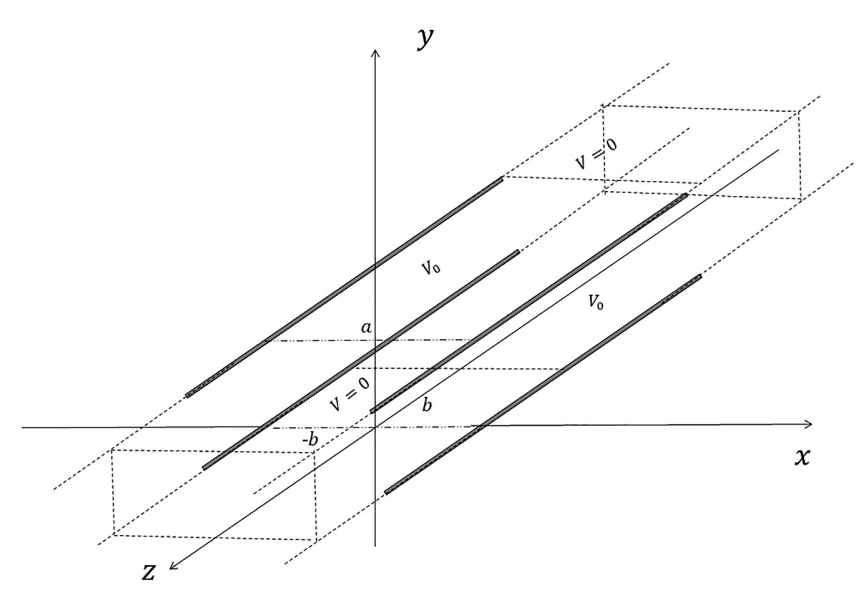

Figura 1: Tubo retangular infinito.
Dessa forma, podemos perceber, da equação (52), que a dependencia na variável $x$ é igual a dependencia em $y$, a menos de uma constante. Portanto, resolvemos a 52 como duas equações diferenciais ordinárias de segunda ordem, obtendo as soluções

$$
\begin{aligned}
& X(x)=A e^{k x}+B e^{-k x} \\
& Y(y)=C \sin (k y)+D \cos (k y)
\end{aligned}
$$

Sendo assim, a equação de Laplace em duas dimensões possui solução

$$
V(x, y)=\left[A e^{k x}+B e^{-k x}\right][C \sin (k y)+D \cos (k y)] .
$$

Para que (55) satisfaça as condições de contorno $V(x, 0)$ e $V(x, a)$, temos que $D=0$ e $k=n \pi / a$, onde $n \in \mathbb{N}$. Logo, temos

$$
V_{n}(x, y)=\left[A_{n} e^{(n \pi x / a)}+B_{n} e^{-(n \pi x / a)}\right] \sin \left(\frac{n \pi y}{a}\right) .
$$

As condições $V_{n}(b, y)=V_{n}(-b, y)$ implicam que a dependência em $x$ está associada uma função par, o que pode ser feito por meio da relação $e^{(n \pi x / a)}+e^{-(n \pi x / a)}=$ $2 \cosh (n \pi x / a) \operatorname{com} A_{n}=B_{n}$. Como todos os $V_{n}(x, y)$ satisfazem as condições iniciais e a equação de Laplace, então podemos escrever uma solução geral como a seguinte soma de todos eles [6]

$$
V(x, y)=\sum_{n=1}^{\infty} A_{n} \cosh \left(\frac{n \pi x}{a}\right) \sin \left(\frac{n \pi y}{a}\right) .
$$

Nesta conjectura, tomando $V(b, y)=\varphi(y)$, temos

$$
\varphi(y)=\sum_{n=1}^{\infty} A_{n} \cosh \left(\frac{n \pi b}{a}\right) \sin \left(\frac{n \pi y}{a}\right) .
$$

Note que $\varphi(y)$ apresenta uma expansão de Fourier na forma

$$
f\left(y^{\prime}\right)=\sum_{n=1}^{\infty} b_{n} \sin \left(n y^{\prime}\right)
$$

onde

$$
b_{n}=\frac{2}{\pi} \int_{0}^{\pi} \sin \left(n y^{\prime}\right) d y^{\prime}
$$

Dessa forma, $A_{n}$ é um coeficiente de Fourier o qual é determinado fazendo $y^{\prime} \rightarrow y / a$ e $d y^{\prime} \rightarrow d y / a$ e ajustando os limites de integração,

$$
A_{n}=\frac{2}{a \cosh (n \pi b / a)} \int_{0}^{a} \varphi(y) \sin \left(\frac{n \pi y}{a}\right) d y
$$

Portanto, para $\varphi(y)=V_{0}$, temos que

$$
A_{n}=\frac{4}{n \pi \cosh (n \pi b / a)},
$$

com $n$ sendo ímpar. Portanto, o potencial (56) satisfaz 50 com $A_{n}$ sendo um coeficiente de Fourier dado por (58), 


$$
V(x, y)=\frac{4 V_{0}}{\pi} \sum_{n=0}^{\infty} \frac{\cosh [(2 n+1) \pi x / a]}{(2 n+1) \cosh [(2 n+1) \pi b / a]} \sin \left[\frac{(2 n+1) \pi y}{a}\right],
$$

aqui usamos somente os valores ímpares de $n \rightarrow 2 n+1$. Esse é o mesmo potencial obtido em [3].

Além disso, podemos obter o campo elestrosatático a partir do gradiente negativo do potencial (59),

$$
\vec{E}(x, y)=-4 V_{0} \sum_{n=0}^{\infty}\left\{\frac{\sinh [(2 n+1) x / a]}{a \cosh [(2 n+1) \pi b / a]} \sin \left[\frac{(2 n+1) \pi y}{a}\right] \hat{i}+\frac{\cosh [(2 n+1) x / a]}{a \cosh [(2 n+1) \pi b / a]} \cos \left[\frac{(2 n+1) \pi y}{a}\right] \hat{j}\right\} .
$$

Agora nós vamos utilizar o software Mathematica para representar graficamente o potencial eletrostático e o campo elétrico associado a ele. Para isso, usamos o comando Plot3D para gerar um gráfico do potencial. O comando Plot3D gera gráficos em três dimensões de uma função de duas variáveis reais independentes, como $x$ e $y$ por exemplo. Como o potencial (59) é uma função de duas variáveis reais, podemos representá-lo através desse comando no Mathematica. $\mathrm{O}$ resultado se vê na Figura 2 onde atribuímos os seguintes valores às constantes $V_{0}=a=b=1$. Podemos também representar graficamente um campo vetorial bidimensional através do comando VectorPlot no software Mathematica. $\mathrm{O}$ resultado é visualizado como pequenas setas, representando os vetores naquela posição, distribuem-se ao longo do plano $x y$. Assumindo novamente $V_{0}=a=b=1$, usando o comando VectorPlot para a expressão (60), temos a representação gráfica do campo elétrico na Figura 3

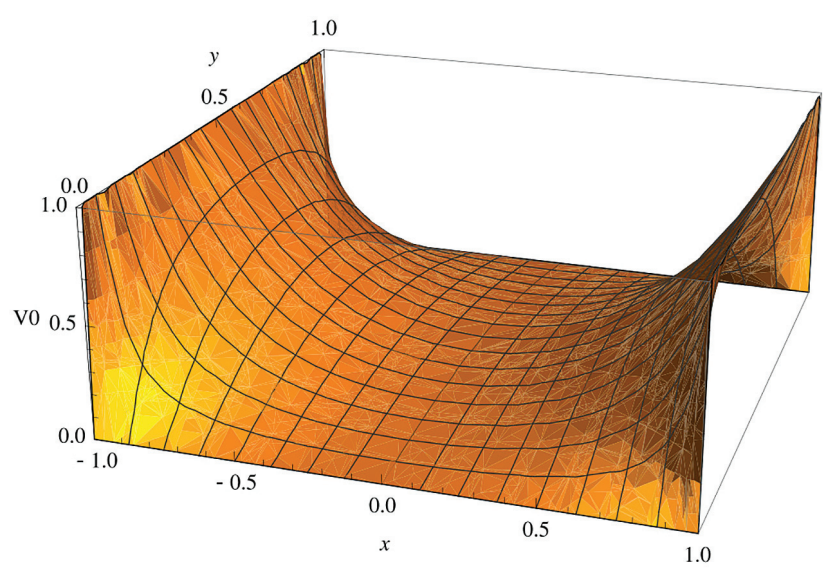

Figura 2: Comportameto do potencial eletrostático (59) com os parâmetros $V_{0}=a=b=c=1$.

Podemos verificar através do gráfico do potencial eletrostático que o mesmo tem um comportamento crescente na direção do módulo crescente de $x$, tendo valor extremo nas bordas de $x= \pm 1$. Isso não significa que exista algum máximo ou mínimo entre os intervalos $x \in(-1,1)$ e $y \in(0,1)$. Temos então uma situação em pleno acordo com o teorema da forma forte do princípio dos máximos e mínimos, o qual diz que uma solução da equação de Laplace com condição de Dirichlet não pode ter máximos ou mínimos locais na região limitada de validade da solução, a não ser que ela seja uma constante numérica, ou seja, que ela permaneça com um valor numérico fixo com a variação das variáveis independentes [8, 13] (Uma função possui máximo local em um ponto quando a primeira deivada, em relação a variável independente, é zero ou não existe derivada (um bico), e a segunda derivada é negativa nesse ponto. Caso a segunda derivada seja positiva, e a prmeira zero, então temos um mínimo local). O leitor não



Figura 3: Esse gráfico representa o campo elétrico associado ao potencial (59) com os mesmos parâmetros da Figura (2). O eixo horizontal representa a coordenada $x$ em metros, que varia de $-1 m$ até $1 m$, e o eixo vertical representa a coordenada $y$ em metros, aqual varia de zero até $1 \mathrm{~m}$.

deve se confundir com as curvas de $V(x, y)$ tomando $y$ constante, na Figura 2, elas não apresentam um mínimo entre $x \in(-1,1)$. Da mesma forma as curvas de $V(x, y)$ tomando $x$ constante, elas não apresentam um máximo entre $y \in(0,1)$. Para esclarecer melhor o que explicamos acima, vamos fazer o caso particular em uma dimensão da equação de Laplace $d^{2} V(x) / d x^{2}=0$ na região de intervalo real $x \in\left[x_{0}, x_{1}\right]$. Integrando duas vezes essa equação, obtemos a seguinte solução $V(x)=c_{1} x+c_{0}$, onde $c_{1}, c_{0} \in \Re$ e $x \in\left[x_{0}, x_{1}\right]$. Essa solução representa um segmento de reta em que 
temos as seguintes possibilidades: (a) a reta é crescente, $c_{1}>0$, e temos um máximo em $x=x_{1}$ e um mínimo em $x=x_{0}$; (b) a reta é decrescente, $c_{1}<0$, e temos um máximo em $x=x_{0}$ e um mínimo em $x=x_{1}$; e finalmente (c) a reta é horizontal, $c_{1}=0$, e a solução $V(x)=c_{0}$ é uma constante real. Vemos então que nos casos (a) e (b) os máximos e mínimos estão nas bordas do intervalo real $\left[x_{0}, x_{1}\right]$, e não podem aparecer no interior desse intervalo. Já no caso (c), podemos ter máximo em qualquer valor do intervalo real $\left[x_{0}, x_{1}\right]$, pois a solução permanece com um valor constante, não importa para qual valor da variável $x$ (ver seção 1.3 página 14 de [14]). Esses conceitos podem ser estendidos para duas ou três dimensões.

O gráfico da Figura 3 mostra a distribuição de vetores representando o campo elétrico 60, onde podemos verificar a maior intensidade do campo para as cores mais claras. Na região $y=0$ o campo aponta para valores decrescente de $y$, e na região $y=1$ o contrário. Para uma partícula teste carregada com carga $Q$, a força que atua sobre ela é dada pela força de Lorentz $\vec{F}=Q \vec{E}+Q \vec{v} \times \vec{B}$. Como não temos campo magnético neste caso, se colocarmos tal partícula na região $y=0$ (ou $y=1$ ), a mesma seria impelida para fora da região do gráfico, por uma força elétrica. Se colocarmos tal partícula no ponto $(-1,0.5)$, a mesma seria impelida na direção $x$, para valores crescente (o contrário para o ponto $(1,0.5)$ ). Assim, em qualquer ponto que a partícula seja colocada, ela sofreria a ação de uma força elétrica, a qual a aceleraria na mesma direção dos vetores da representação no gráfico da Figura (3).

\section{Problema do Potencial para Condições de Contorno de uma Caixa Retangular}

Nesta seção, trataremos do objetivo princial desse artigo que é a aplicação do princípio de superposição da equação de Laplace na resolução do problema de um potencial eletrostático com condições de contorno para uma caixa retangular.

Os livros como 3 6] tratam o problema tridimencional da equação de Laplace através de uma caixa retangular em que somente uma das seis faces está sujeita a um potencial eletrostático diferente de zero, e muitas das vezes constante, e as outras cinco faces aterradas e neutras. Apesar de que esses livros trazem uma forma geral de solução da equação de Laplace em três dimensões, eles não resolvem nenhum caso em que duas ou mais faces de uma caixa retangular estejam sujeitas a um potencial diferente de zero, e as outras com potenciais iguais a zero. Esses livros mostram o princípio de superposição quando consideram a soma das possíveis soluções do potencial geral, mas não mostram explicitamente como resolver um problema um pouco mais geral que o inicial citado aqui. Nossa proposta, diferentes dos livros

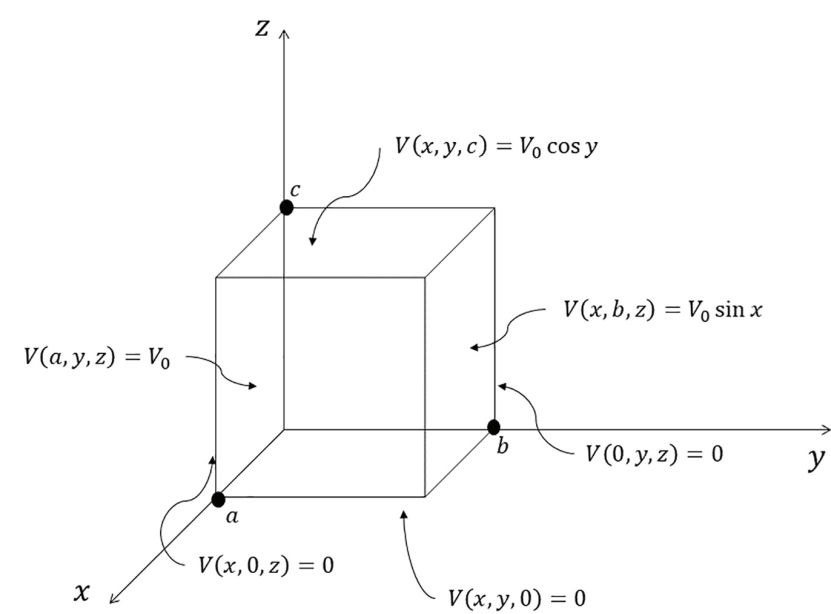

Figura 4: Caixa retangular.

textos, é resolver o caso da equação de Laplace em três dimensões, com condições de contorno em que três faces estejam sujeitas a potenciais diferente de zero, e as outras três faces com potenciais nulo. Também mostrar como obter os coeficientes de Fourier em uma série dupla de Fourier, o que também não é mostrado nos livros textos. O problema analisado aqui foi proposto em um exercício do capítulo 6 do livro [6], número 6.2 , em que se usa uma condição de contorno fictícia, somente para ilustrar como se utiliza o princípio de superposição. Acreditamos que essas condições podem ser físicas, pois podemos sujeitar duas das faces a potenciais independente do tempo entretanto oscilatórios em uma das coordenadas. Também acreditamos que essa escolha se deva a integrabilidade dos coeficientes de Fourier.

Se considerarmos uma caixa condutora retangular, de dimensões $a, b$ e $c$ (ver Figura 44, livre de cargas, em que a face 1 , em $x=a$, está sujeita a um potencial constante $V_{0}$, a face 2 , em $y=b$, sujeita a um potencial $V_{0} \sin (x)$, a face 3 , em $z=c$, sujeita ao potencial $V_{0} \cos (y)$, e todas as faces restantes sujeitas ao potencial nulo. Perguntasse: qual é o potencial eletrostático no interior da caixa? Esse problema é resolvido solucionando-se a equação de Laplace em três dimensões em coordenadas cartesianas. Mas temos que usar um importante método, que consiste em separar a resolução em três etapas. Primeiro considerando o problama de uma caixa, em que a face 1 esteja sujeita ao potencial cosntate $V_{0}$, e as demais faces sujeitas ao potencial nulo; segundo considerando o problem de uma caixa em que a face 2 está sujeita ao potencial $V_{0} \sin (x)$, e as demais faces sujeitas ao potencial nulo; e por fim o terceiro problema, em que a face 3 está sujeita ao potencial $V_{0} \cos (y)$, e todas as demais faces sujeitas ao potencial nulo. Ao final, a solução da equação de Laplace para as condições de contorno geral, usando o princípio de superposição, será a soma das três soluções obtidas em cada etapa. 
Vamos então tomar as seguintes condições de contorno:

$$
\begin{cases}V(0, y, z)=0 & V(a, y, z)=V_{0} \\ V(x, 0, z)=0 & V(x, b, z)=V_{0} \sin (x) \\ V(x, y, 0)=0 & V(x, y, c)=V_{0} \cos (y)\end{cases}
$$

A solução geral da equaçao de Laplace apresentada na subseção 2.2, a qual é a soma das soluções particulares dadas pelas equações $(35),(36),(37),(38)$ e $(39)$, tomando o exemplo da caixa acima, fica impraticável de discernir quais constantes devem ser iguais a zero e quais devem ser mantidas, para depois serem encontardas pelo coeficiente de Fourier, caso se considere simultâneamente todas as condições de contorno diferente de zero. Portanto, a expressão final deve ser obtida por meio do princípio de superposição, no qual o problema será dividido em três etapas, onde as soluções serão atribuídas a cada face da superfície com potencial diferente de zero, considerando os potenciais das outras faces nulos. Isto posto, as novas condições de contorno tornam-se:

$$
\begin{aligned}
& \begin{cases}V_{1}(0, y, z)=0 & V_{1}(a, y, z)=V_{0} \\
V_{1}(x, 0, z)=0 & V_{1}(x, b, z)=0 \\
V_{1}(x, y, 0)=0 & V_{1}(x, y, c)=0\end{cases} \\
& \begin{cases}V_{2}(0, y, z)=0 & V_{2}(a, y, z)=0 \\
V_{2}(x, 0, z)=0 & V_{2}(x, b, z)=V_{0} \sin (x) \\
V_{2}(x, y, 0)=0 & V_{2}(x, y, c)=0\end{cases} \\
& \begin{cases}V_{3}(0, y, z)=0 & V_{3}(a, y, z)=0 \\
V_{3}(x, 0, z)=0 & V_{3}(x, b, z)=0 \\
V_{3}(x, y, 0)=0 & V_{3}(x, y, c)=V_{0} \cos (y)\end{cases}
\end{aligned}
$$

Note que a soma dos três potenciais, $V_{1}, V_{2}$ e $V_{3}$, fornece o resultado para (61). No entanto, uma certa independência é apresentado uma vez que a relação (31) não é a mesma entre eles. Portanto, existem $k, l$ e $\lambda$ diferentes à cada contorno, definidas no respectivo potencial associado.

Para os contornos $\mathcal{V}(0, y, z)=0$ e $\mathcal{V}(x, 0, z)=0$, a equação 40 só os satisfaz se $c_{0,0}=c_{k, 0}=c_{k, k}=c_{k, l}=$ $0, c_{0, l}=-d_{0, l}$ e, $\forall k$ e $l, a_{k, l}=0$. Logo,

$$
\begin{aligned}
\mathcal{V}(x, y, z)= & \mathcal{C}_{1} x y\left(f_{0,0}+g_{0,0} z\right) \\
& +\mathcal{C}_{2} x \sin k y\left(f_{k, 0} e^{k z}+g_{k, 0} e^{-k z}\right) \\
& +\mathcal{C}_{3} \sin l x \sinh l y\left(f_{0,0}+g_{0,0} z\right) \\
& +\mathcal{C}_{4} y \sin k x\left(f_{k, k} e^{k z}+g_{k, k} e^{-k z}\right) \\
& +\mathcal{C}_{5} \sin l x \sin \lambda y\left(f_{k, l} e^{k z}+g_{k, l} e^{-k z}\right)
\end{aligned}
$$

Na expressão $65, \mathcal{C}$ é o produto de constantes, para simplificar a notação; e $\mathcal{V}$ será usado como ponto de partida uma vez que é válido para qualquer condição de contorno na qual o potencial é nulo em planos gerados no espaço onde $x, y$ ou $z$ são nulos.

Da equação (65), os contornos $V_{1}(x, b, z)=0 \mathrm{e}$ $V_{1}(x, y, 0)=0$ implica $\mathcal{C}_{1}=\mathcal{C}_{2}=\mathcal{C}_{3}=\mathcal{C}_{4}=0$, $\lambda_{1}=n \pi y / b$ e $f_{k, l}=-g_{k, l}$. Assim,

$$
V_{1}(x, y, z)=\mathcal{C}_{5} \sin \left(l_{1} x\right) \sin \left(\frac{n \pi y}{b}\right) f_{k_{1}, l_{1}}\left[e^{k_{1} z}-e^{-k_{1} z}\right] .
$$

Note que $k_{1}=n \pi / b$ seria uma possibilidade ao invés de $\mathcal{C}_{2}=0$. No entanto, a segunda opção é a correta porque $V_{1}(x, y, c)=0$ é satisfeita se $k_{1}=i \gamma \pi / c, f_{k_{1}, l_{1}}=$ $\mathcal{C}^{\prime} / 2 i$, sendo $\gamma=m \pi / c$, onde $m \in \mathbb{N}$. Temos,

$$
\begin{aligned}
V_{1}(x, y, z) & =\mathcal{C}_{5} \sin \left(l_{1} x\right) \sin \left(\frac{n \pi y}{b}\right) f_{k_{1}, l_{1}} \sin \left(\frac{m \pi z}{c}\right) \\
& =\mathcal{C}_{5} \sin \left(l_{1} x\right) \sin \left(\frac{n \pi y}{b}\right) \sin \left(\frac{m \pi z}{c}\right),
\end{aligned}
$$

$\mathrm{Ou}$

$$
V_{1}(x, y, z)=\sum_{n, m=1}^{\infty} \mathcal{C}_{5} \sin \left(l_{1} x\right) \sin \left(\frac{n \pi y}{b}\right) \sin \left(\frac{m \pi z}{c}\right) .
$$

As constantes $k_{1}$ e $l_{1}$ estão relacionados pela (31), e são imaginários puros

$$
\begin{aligned}
l_{1} & =\sqrt{\left[\frac{i \pi m}{c}\right]^{2}-\left[\frac{\pi n}{b}\right]^{2}} \\
& =i \sqrt{\left[\frac{\pi m}{c}\right]^{2}+\left[\frac{\pi n}{b}\right]^{2}}=i \bar{l}_{n, m}
\end{aligned}
$$

Podemos facilmente voltar a um resultado real se tivermos uma constate imaginária pura do tipo $\mathcal{C}^{\prime}=\mathcal{C} / i$. Logo,

$V_{1}(x, y, z)=\sum_{n, m=1}^{\infty} \mathcal{C}_{5} \sinh \left(\bar{l}_{n, m} x\right) \sin \left(\frac{n \pi y}{b}\right) \sin \left(\frac{m \pi z}{c}\right)$

Por fim, consideramos o contorno arbitrário $V_{1}$ $(a, y, z)=f(y, z)$, uma vez que $\mathcal{A}_{n, m}=\mathcal{C}_{5} \sinh \left(\bar{l}_{1} x\right)$ recaímos na forma do coeficiente da série de Fourier em duas dimensões, equação 49,

$$
\mathcal{A}_{n, m}=\frac{16 V_{0}}{\sinh \bar{l}_{n, m} a(2 n+1)(2 m+1) \pi^{2}}
$$

Portanto, o potencial do contorno 62 , é dado pela expressão 68.

$$
V_{1}(x, y, z)=\frac{16 V_{0}}{\pi^{2}} \sum_{n, m=0}^{\infty} \frac{\sinh \left(\bar{l}_{n, m} x\right)}{\sinh \left(\bar{l}_{n, m} a\right)(2 n+1)(2 m+1)} \sin \left[\frac{(2 n+1) \pi y}{b}\right] \sin \left[\frac{(2 m+1) \pi z}{c}\right]
$$


sendo,

$$
\bar{l}_{n, m}=\sqrt{\left[\frac{\pi(2 m+1)}{c}\right]^{2}+\left[\frac{\pi(2 n+1)}{b}\right]^{2}}
$$

A segunda etapa do problema tem uma grande semelhança com a primeira. Note que a constante complexa é um truque que sempre será usado para condições de contorno $\mathcal{V}(a, y, z)$ e $\mathcal{V}(x, b, z)$ diferentes de zero quando todas as outras forem zero. O resultado final difere nas variáveis, é certo que dois termos vão ser funções seno (cujo argumento terá um índice, par ou não), e na contribuição constante advinda da série de Fourier. Isso fica claro quando aplicamos as condições de contorno $V_{2}(x, y, 0)=0, V_{2}(x, y, a)=0$ e $V_{2}(a, y, z)=0$ na expressão 65 , o resultado será que $\mathcal{C}_{1}=\mathcal{C}_{2}=\mathcal{C}_{3}=$ $\mathcal{C}_{4}=0, f_{k_{2}, l_{2}}=-g_{k_{2}, l_{2}}, k_{2}=i \gamma$, a constante sendo imaginário puro $f_{k_{2}, l_{2}}=\mathcal{C}^{\prime} / 2 i$ e $\gamma=m \pi / c$, reobtendo seno na variável $z$. Porém, dessa vez $l_{2}=n \pi / a$. Assim,

$$
V_{2}(x, y, z)=\sum_{n, m=1}^{\infty} \mathcal{C}_{5} \sin \left(\lambda_{2} y\right) \sin \left(\frac{n \pi x}{a}\right) \sin \left(\frac{m \pi z}{c}\right)
$$

sendo,

$$
\lambda_{2}=\sqrt{\left[\frac{i \pi m}{c}\right]^{2}-\left[\frac{\pi n}{b}\right]^{2}}
$$

$$
\begin{aligned}
& =i \sqrt{\left[\frac{\pi m}{c}\right]^{2}+\left[\frac{\pi n}{b}\right]^{2}} \\
& =i \bar{\lambda}_{n, m}
\end{aligned}
$$

Para voltar ao resultado real, faz-se uma constante do tipo $\mathcal{C}^{\prime}=\mathcal{C} / 2 i$. Portanto,

$$
V_{2}(x, y, z)=\mathcal{C}_{5} \sinh \left(\bar{\lambda}_{2} y\right) \sin \left(\frac{n \pi x}{a}\right) \sin \left(\frac{m \pi z}{c}\right)
$$

$\mathrm{ou}$

$$
V_{2}(x, y, z)=\sum_{n, m=1}^{\infty} \mathcal{C}_{5} \sinh \left(\bar{\lambda}_{2} y\right) \sin \left(\frac{n \pi x}{a}\right) \sin \left(\frac{m \pi z}{c}\right) .
$$

Um ponto interessante de se notar, embora $V_{1}$ e $V_{2}$ sejam soluções independentes, pode-se definir $\lambda_{2}$ e $k_{1}$ nos mesmo índices, escolha a qual reduz o custo computacional de plotar essas expressões.

O coeficiente de Fourier equação 49, com $x^{\prime}=$ $n \pi x / a, y^{\prime}=n \pi y / b$ e $f(x, z)=V_{0} \sin x$, é determinado em

$$
\mathcal{A}_{n, m}=\frac{8 V_{0} n(-1)^{n} \sin a}{\sinh \bar{\lambda}_{n, m} a\left(a^{2}-\pi^{2} n^{2}\right)(2 m+1)} .
$$

Por conseguinte, o termo $\mathcal{C}_{5}$ é equivalente à 71 para que $V_{2}(x, b, z)=V_{0} \sin x$ seja satisfeito, resultando em

$$
V_{2}(x, y, z)=8 V_{0} \sum_{n, m=0}^{\infty} \frac{\sinh \left(\bar{\lambda}_{n, m} y\right) n(-1)^{n} \sin a}{\sinh \left(\bar{\lambda}_{n, m} b\right)\left(a^{2}-\pi^{2} n^{2}\right)(2 m+1)} \sin \left(\frac{n \pi x}{b}\right) \sin \left[\frac{(2 m+1) \pi z}{c}\right]
$$

sendo

$$
\bar{\lambda}_{n, m}=\sqrt{\left[\frac{\pi(2 m+1)}{c}\right]^{2}+\left[\frac{\pi n}{a}\right]^{2}}
$$

Para a última etapa, o método é mais simples e a constante complexa não é usada. As condições de contorno $V_{3}(x, y, z)=0, V_{3}(a, y, z)=0$ e $V_{3}(x, b, z)=0$ podem ser imediatamente substituídas na (65), onde constata-se que $\mathcal{C}_{1}=\mathcal{C}_{2}=\mathcal{C}_{3}=\mathcal{C}_{4}=0, l_{3}=n \pi x / a, \lambda_{3}=m \pi y / b$ e $f_{k, l}=-g_{k, l}$, obtendo

$$
V_{3}(x, y, z)=\sum_{n, m=1}^{\infty} \mathcal{C}_{5} \sinh \left(k_{n, m} z\right) \sin \left(\frac{n \pi x}{a}\right) \sin \left(\frac{m \pi y}{b}\right) .
$$

O coeficiente $A_{n, m}$ é determinado, para a ultima condição de contorno, em,

$$
\mathcal{A}_{n, m}=\frac{8 V_{0} m\left[\cos b(-1)^{m}-1\right]}{\sinh k_{n, m} c\left(b^{2}-\pi^{2} m^{2}\right)(2 n+1)}
$$

Por fim,

$$
V_{3}(x, y, z)=8 V_{0} \sum_{n, m=0}^{\infty} \frac{8 V_{0} m\left[\cos b(-1)^{m}-1\right] \sinh \left(k_{n, m} z\right)}{\sinh k_{n, m} c\left(b^{2}-\pi^{2} m^{2}\right)(2 n+1)} \sin \left[\frac{(2 n+1) \pi x}{a}\right] \sin \left(\frac{m \pi y}{b}\right),
$$

sendo

$$
k_{n, m}=\sqrt{\left[\frac{\pi(2 n+1)}{c}\right]^{2}+\left[\frac{\pi m}{a}\right]^{2}}
$$


Finalmente, o potencial gerado pelas condições de contorno 61 é dado pela soma de (68), (72) e 775, em conformidade com o teorema da superposição. O resultado final é o seguinte potencial

$$
\begin{aligned}
V(x, y, z)= & 8 V_{0} \sum_{n, m=0}^{\infty}\left\{\frac{2 \sinh \left(\bar{l}_{n, m} x\right)}{\sinh \left(\bar{l}_{n, m} a\right)(2 n+1)(2 m+1) \pi^{2}} \sin \left[\frac{(2 n+1) \pi y}{b}\right] \sin \left[\frac{(2 m+1) \pi z}{c}\right]\right. \\
& +\frac{\sinh \left(\bar{\lambda}_{n, m} y\right) n(-1)^{n} \sin a}{\sinh \left(\bar{\lambda}_{n, m} b\right)(2 n+1)(2 m+1)} \sin \left[\frac{n \pi x}{a}\right] \sin \left[\frac{(2 m+1) \pi z}{c}\right] \\
& \left.+\frac{m\left[(-1)^{m} \cos b-1\right] \sinh \left(k_{n, m} z\right)}{\sinh \left(k_{n, m} c\right)\left(b^{2}-\pi^{2} m^{2}\right)(2 n+1)} \sin \left[\frac{(2 n+1) \pi x}{a}\right] \sin \left[\frac{m \pi y}{b}\right]\right\}
\end{aligned}
$$

Como consequência, o campo elétrico pode ser calculado imediatante através do seu gradiente negativo, cujas componentes são

$$
\begin{aligned}
E_{x}(x, y, z)= & -8 V_{0} \sum_{n, m=0}^{\infty}\left\{\frac{2 \bar{l}_{n, m} \cosh \left(\bar{l}_{n, m} x\right)}{\sinh \left(\bar{l}_{n, m} a\right)(2 n+1)(2 m+1) \pi^{2}} \sin \left[\frac{(2 n+1) \pi y}{b}\right] \sin \left[\frac{(2 m+1) \pi z}{c}\right]\right. \\
& +\frac{n^{2}(-1)^{n+1} \pi \sinh \left(\bar{\lambda}_{n, m} y\right) \sin a}{a \sinh \left(\bar{\lambda}_{n, m} b\right)(2 n+1)(2 m+1)} \cos \left[\frac{n \pi x}{a}\right] \sin \left[\frac{(2 m+1) \pi z}{c}\right] \\
& \left.+\frac{m \pi(2 n+1)\left[(-1)^{m} \cos b-1\right] \sinh \left(k_{n, m} z\right)}{a \sinh \left(k_{n, m} c\right)\left(b^{2}-\pi^{2} m^{2}\right)(2 n+1)} \cos \left[\frac{(2 n+1) \pi x}{a}\right] \sin \left[\frac{m \pi y}{b}\right]\right\} \\
E_{y}(x, y, z)= & -8 V_{0} \sum_{n, m=0}^{\infty}\left\{\frac{(4 n+2) \sinh \left(\bar{l}_{n, m} x\right)}{b \sinh \left(\bar{l}_{n, m} a\right)(2 n+1)(2 m+1) \pi} \cos \left[\frac{(2 n+1) \pi y}{b}\right] \sin \left[\frac{(2 m+1) \pi z}{c}\right]\right. \\
& +\frac{n(-1)^{n} \bar{\lambda}_{n, m} \cosh \left(\bar{\lambda}_{n, m} y\right) \sin a}{\sinh \left(\bar{\lambda}_{n, m} b\right)(2 n+1)(2 m+1)} \sin \left[\frac{n \pi x}{a}\right] \sin \left[\frac{(2 m+1) \pi z}{c}\right] \\
& \left.+\frac{\pi m^{2}\left[(-1)^{m} \cos b-1\right] \sinh \left(k_{n, m} z\right)}{b \sinh \left(k_{n, m} c\right)\left(b^{2}-\pi^{2} m^{2}\right)(2 n+1)} \sin \left[\frac{(2 n+1) \pi x}{a}\right] \cos \left[\frac{m \pi y}{b}\right]\right\} \\
& \left.+\frac{k_{n, m} m\left[(-1)^{m} \cos b-1\right] \cosh \left(k_{n, m} z\right)}{\sinh \left(k_{n, m} c\right)\left(b^{2}-\pi^{2} m^{2}\right)(2 n+1)} \sin \left[\frac{(2 n+1) \pi x}{a}\right] \sin \left[\frac{m \pi y}{b}\right]\right\} \\
& +\frac{n(-1)^{n}(2 m+1) \pi \sinh \left(\bar{\lambda}_{n, m} y\right) \sin a}{c \sinh \left(\bar{\lambda}_{n, m} b\right)(2 n+1)(2 m+1)} \sin \left[\frac{n \pi x}{a}\right] \cos \left[\frac{(2 m+1) \pi z}{c}\right] \\
E_{z}(x, y, z)= & 8 V_{0} \sum_{n, m=0}\left\{\frac{(4 m+2) \sinh \left(\bar{l}_{n, m} x\right)}{\sinh \left(\bar{l}_{n, m} a\right)(2 n+1)(2 m+1) \pi} \sin \left[\frac{(2 n+1) \pi y}{b}\right] \cos \left[\frac{(2 m+1) \pi z}{c}\right]\right.
\end{aligned}
$$

Tanto o potencial geral (77), quanto o campo elétrico associado a ele, são resultados inéditos obtidos apenas aqui nesse trabalho. Como mencionado, o livro [6] apenas sugere esse problema como um exercício, sem mostrar a solução.

\subsection{Analise do potencial eletrostático em uma caixa}

Nesta seção faremos algumas representações que possibilitem uma visualização da solução do potencial eletrostático (77). O comando CountourPlot3D do software Mathematica é usado para gerar superfícies de contorno para uma determinada função de três variáveis reais. Por exemplo, se tivermos o potencial eletrostático $V(x, y, z)$, podemos criar superfícies $V(x, y, z)=k_{i}$, com $k_{i}$ constantes reais, e assim vizualizar como as superfícies equipotenciais se distribuem. Se $V(x, y, z)=x^{2}+y^{2}$, então o comando ContourPlot3D, para os intervalos $x, y, z \in$ $(-1,1)$, resulta em alguns cilindros concêntricos no eixo $z$, de várias cores. Para gerenciar o valor numérico crescente do potencial, usa-se o comando ColorFunction $->$ "Rainbow"inserido dentro do comando ContourPlot3D, isso gera cores do arco-íres começando com violeta e terminando com o vermelho. Então podemos saber quais as superfícies equipotenciais têm o valor numérico maior. $O$ resultado pode ser visto na Figura 5 , onde o cilindro violeta é o de menor potencial, o verde o mediano, e 


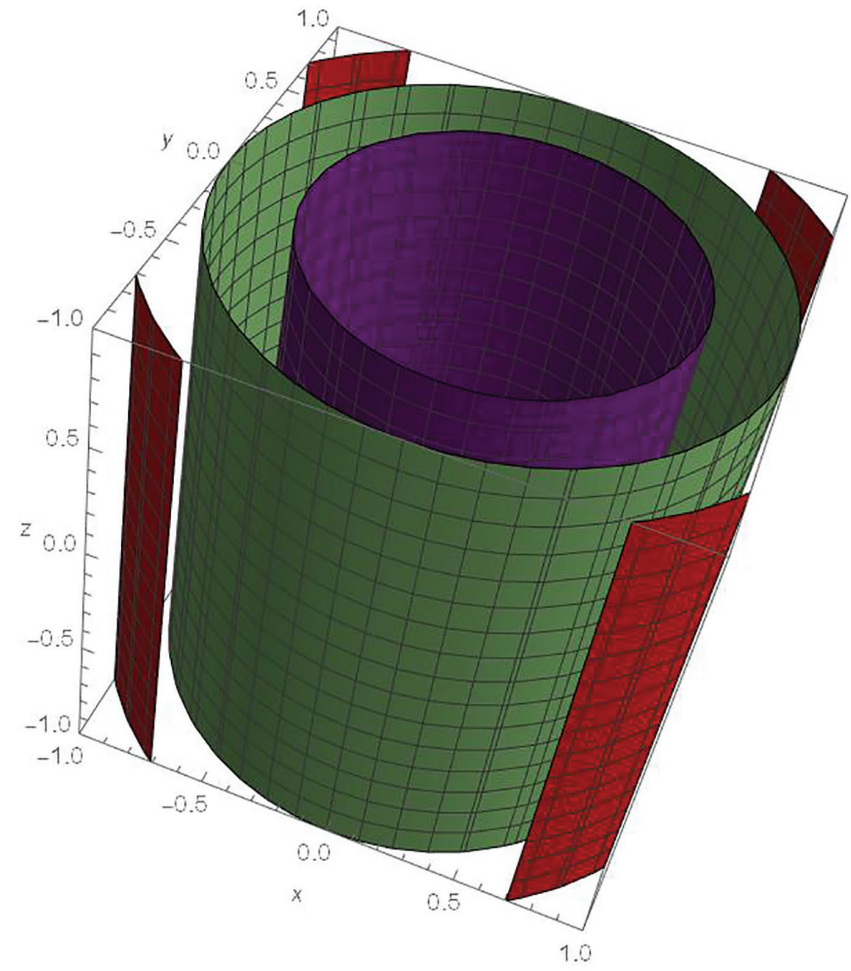

Figura 5: Figura gerada com o comando ContourPlot3D e ColorFunction - $>$ "Rainbow" para $V(x, y)=x^{2}+y^{2}$.

o vermelho, onde aparece apenas partes dele, é o de maior potencial. Se tivermos $V(x, y, z)=x^{2}+y^{2}+z^{2}$, escolhendo os intervalos $x, y, z \in(-1,1)$, o comando ContourPlot3D resulta em algumas superfícies esféricas cortadas concêntricas de cores violeta, verde e vermelha, conforme visto na Figura 6 Então podemos ter ao menos alguma visualização para a solução do potencial (77), usando esse comando.

A solução 77) descreve o comportamento do potencial elétrico gerado no interior de uma caixa sujeito às condições de contorno 61), onde uma superfície é gerada de acordo com a escolha dos parâmetros. Definindo $V_{0}=a=b=c=1$, podemos obter o contorno do potencial gerado em uma caixa de um metro cúbico a partir do software Mathematica, utilizando o comando ContourPlot3D, como mostra a Figura 7

A Figura 7 mostra apenas uma das superfícies equipotenciais. Essa superfície encontra-se completamente no intervalo $x \in(0.5,1)$. Isso se dá devido o menor valor escolhido para o potencial, gerar uma superfície que chegue apenas até o meio da coordenada $x$. Mas isso não significa que o potencial $V(x, y, z)$, dado em (77), não seja definido para valores de $x<0.5$, ou mesmo que ele não exista para esses valores. Tomamos o exemplo em que $x=0.25, y=z=0.7$, então o valor numérico do potencial eletrostático é $V(0.25,0.7,0.7)=0.475386$, para $n=m=1000$ termos da série. Então, a superfície gerada para esse valor do potencial, está representada a baixo da azulada, isso vai ficar evidente logo a frente. Essa figura traz outras



Figura 6: Figura gerada com o comando ContourPlot3D e ColorFunction - > "Rainbow" para $V(x, y, z)=x^{2}+y^{2}+z^{2}$.



Figura 7: Contorno da solução (77), para $m$ e $n$ no intervalo $[0,100]$, no interior de uma caixa de $1 \mathrm{~m}^{3}$.

superfícies equipotenciais encobertas. Então devemos representar a mesma figura, com superfícies cortadas para o intervalode de valores de $y \in(0,0.5)$. Assim poderemos ver as outras superfícies equipotenciais que estão abaixo daquela de cor azulada. Fazemos isso na Figura 8. Essa figura foi gerada pelos comandos ContourPlot3D $[V(x, y, z),\{x, 0,1\},\{y, 0.5,1\},\{z, 0,1\}$, AxesLabel $->\{x, y, z\}$, PlotRange - $>\{\{0,1\}$, $\{0,1\},\{0,1\}\}$, Mesh $->\{5,5\}$, Contours $->\{\{V 1$, Red $\},\{V 2$, Yellow $\},\{V 3$, Green $\},\{V 4$, Blue $\},\{V 5$, 


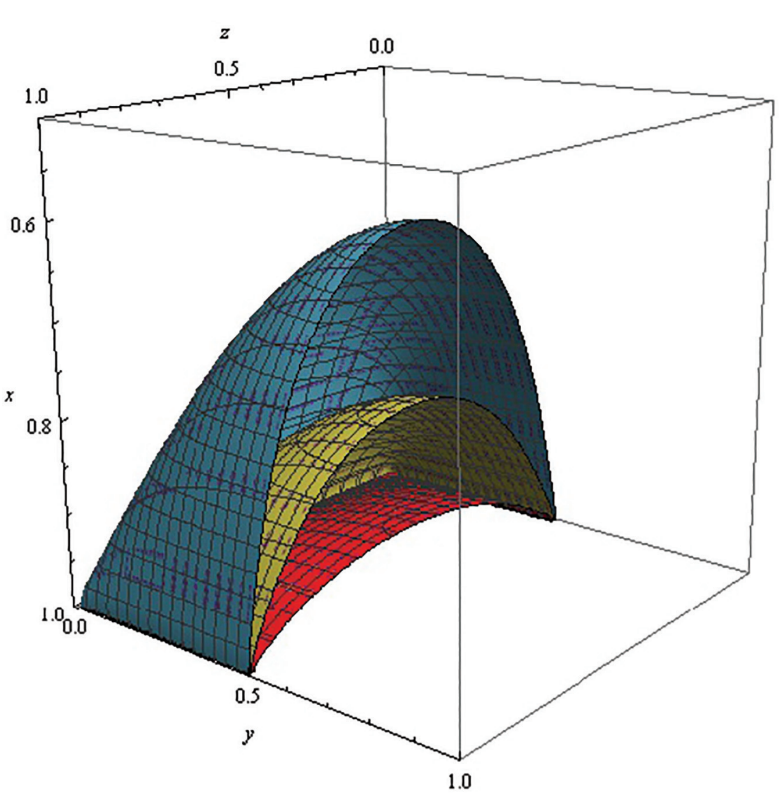

Figura 8: Contorno da solução (77), com $x, y, z, m$ e $n$ variando nos intervalos $[0,1],[1 / 2,1],[0,1],[0,100]$ e $[0,100]$, respectivamente, no interior de uma caixa de $1 \mathrm{~m}^{3}$.

Purple\}\}], onde nós temos $\{V(0.99,0.9,0.9)=V 1=$ $9.051245835270679, V(0.7,0.7,0.7)=V 2=3.07194783$ $29945384, V(0.5,0.5,0.5)=V 3=1.7331192224744325$ $V(0.5,0.5,0.1)=V 4=0.5743612932820764, V(0.1,0.5$, $0.3)=V 5=0.15691167564166233\}$, onde os potenciais foram obtidos para $m=n=1000$. Agora podemos ver claramente que existe um crescimento no valor numérico do potencial, da superfície vermelha para a superfície púrpura, onde temos os valores $V 1>V 2>V 3>$ $V 4>V 5$ para as superfícies vermelha, amarela, verde, azul e púrpura, respectivamente. As superfícies podem preencher toda caixa retangular.

Podemos nos perguntar se as superfícies equipotenciais ainda se distribuem da mesma forma se o volume da caixa retangular aumentar. Considerando dimensões $a=b=c=2 m$, e mantendo $V_{0}=1 V$, comparamos as superfícies equipotenciais dos dois casos, o anterior com essas novas dimensões, na Figura 9 Então nos parece que aumentando as dimensões da caixa, as superfícies equipotenciais mantém um mesmo padrão. Nós não podemos extrair dessas figuras do contorno do potencial eletrostático se o mesmo tem algum máximo ou mínimo. Para fazer isso seria necessário o estudo de derivadas de séries de Fourier, o que foge do objetivo desse estudo feito aqui. Mas ainda podemos garantir, pelo teorema da forma forte de máximos e mínimos, que a solução 777 do potencial eletrostático não possui nem mínimo nem máximo em todo o domínio da solução.

\subsection{Campo elétrico associado ao potencial}

O campo elétrico associado ao potencial (77), muda em pontos distintos do interior da caixa, hora apontando para dentro, hora apontando para fora, devido ao caráter

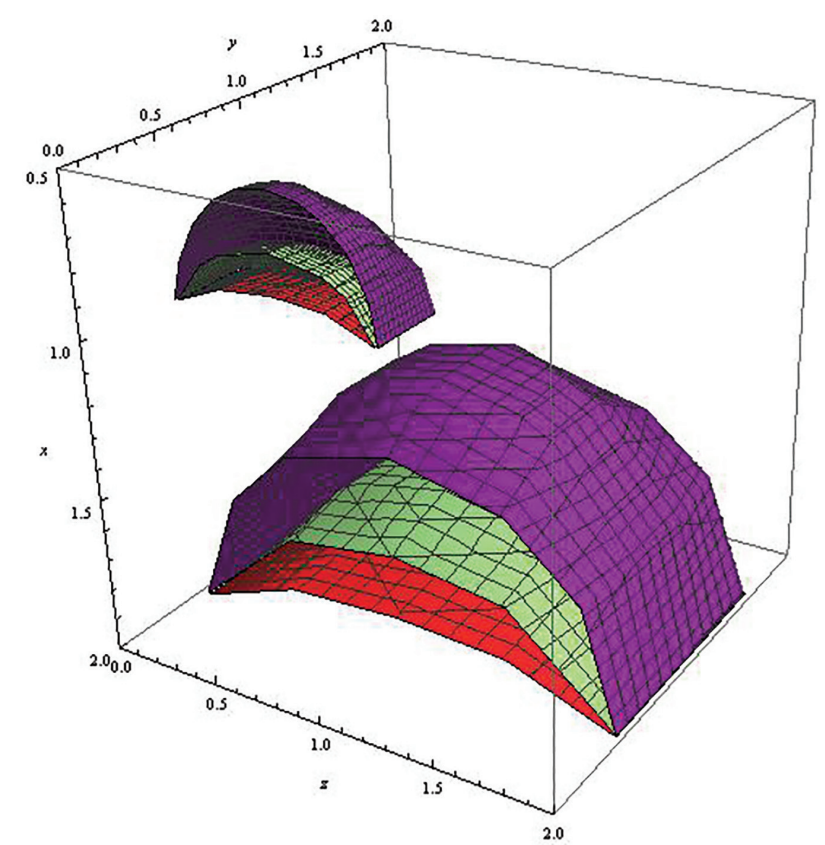

Figura 9: Comparação dos contornos da solução (77) para $a=$ $b=c=1 m$ e $a=b=c=2 m$, ambas com $V_{0}=1 V$ e uma caixa de $8 \mathrm{~m}^{3}$.

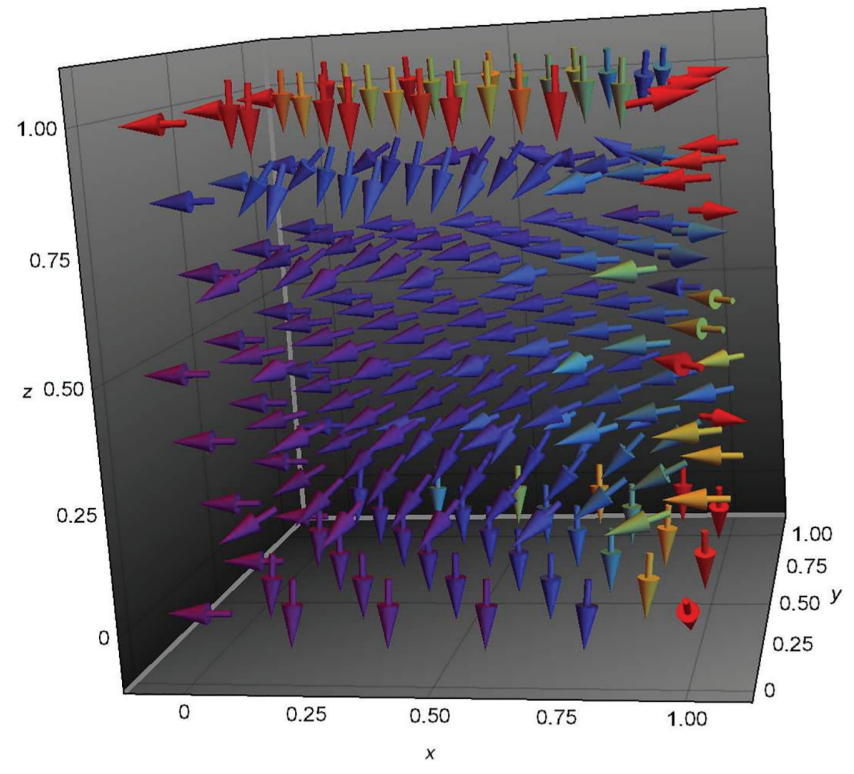

Figura 10: Campo elétrico associado ao potencial (77) com os mesmos parâmetros da Figura (7).

senoidal ou cossenoidal das componentes. Todo interior da caixa é ocupado, porém os pontos de maior intensidade estão no lado em que o potencial corresponde a $V_{0}$ (verificar a Figura 8 onde o maior valor do potencial é representado pela superfície vermelha), como mostra a Figura 10 .

O campo eletrostático da Figura 10 foi gerado no software Mathematica utilizando o comando VectorPlot3D. Podemos ver que os vetores estão preferencialemente 
dirigidos da face $x=a$ para a face $x=0$. Podemos retirar a informação da Figura 8 , onde o crescimento do potencial se dá da face $x=0$ para a face $x=a=1$, logo o gradiente tem a mesma direção. Como o campo elétrico é menos o gradiente do potencial, então o campo elétrico aponta preferencialmente para a mesma direção de menos o gradiente de $V(x, y, z)$, tendo sua intensidade maior nas faces $x=a, y=b$ e $z=c$. Podemos ver que na face $x=a$ os vetores estão em vermelho, intensidade maior, e progressivamente se tornam violeta, de intensidade menor. A verificação do arranjo de vetores do campo elétrico associado a essa solução pode ser feito conforme descrito para a Figura 3.

\section{Aplicação do Potencial Eletrostático no Tratamento de Ondas}

Um dos pontos interessantes de analisar do problema para um potencial no interior de uma caixa retangular é que o campo eletrostático resultante, muitas vezes, pode ser utilizado como parte da solução da equação de Helmholtz; a qual possui um termo espacial e outro temporal. Portanto, é possível controlar a configuração dos campos de tal forma que problemas de potenciais no interior de uma caixa retangular se tornem uma aplicação direta em guias de ondas retangulares e cavidades ressonantes; este ultimo possui ainda uma vantagem particular de substituir os circuitos ressonantes $R L C$, com oscilações forçada e amortecida, os quais não possuem uma precisão confiável devido a perda de energia por radiação que ocorre em frequências acima de $300 \mathrm{MHz}$.

\subsection{Ondas propagando-se no interior de uma caixa retangular}

Foi mostrado na seção 4 a obtenção de um potencial advindo da solução da equação de Laplace, associado a um campo eletrostático, no interior de uma caixa retangular. Note, porém, que o termo $V_{0}$, das condições de contornos (61), é um termo uniforme no espaço e pode ser substituído por uma função do tempo na forma $V(t)=V_{0} e^{-i \omega t}$. Nesse caso, o potencial no instante de tempo $t=0$ é o mesmo que (77), podendo oscilar nos instantes seguintes. Evidentemente, esse problema deixa de ser eletrostático, onde campos elétricos e magnéticos passam a ser descrito pelas equações de Maxwell. Vamos nos restringir a um meio na ausência de fontes de correntes; se considerarmos uma corrente que varia no tempo percorrendo um material condutor, o efeito películas, ou efeito Kelvin, teria que ser levado em consideração; esses efeitos implicam no aparecimento de uma equação de Helmholtz para a densidade de corrente, para a qual vale a Lei de Ohm $\vec{J}=\sigma \vec{E}$ (os trabalhos [15, 16] levam em consideração essa corrente e fazem uma abordagem ao efeito pelicular). Portanto, os campos são descritos por
[3, 5] 7 ]

$$
\begin{gathered}
\nabla \cdot \overrightarrow{\mathcal{E}}=0 \\
\nabla \cdot \overrightarrow{\mathcal{B}}=0 \\
\nabla \times \overrightarrow{\mathcal{E}}=-\frac{\partial \overrightarrow{\mathcal{B}}}{\partial t} \\
\nabla \times \overrightarrow{\mathcal{B}}=\mu_{0} \epsilon_{0} \frac{\partial \overrightarrow{\mathcal{E}}}{\partial t}
\end{gathered}
$$

Da Lei de Faraday, equação $79 \mathrm{c}$, e da Lei de Ampère, equação $79 \mathrm{~d}$, obtemos a equação de onda para os campos elétrico

$$
\nabla^{2} \overrightarrow{\mathcal{E}}-\frac{1}{c^{2}} \frac{\partial^{2} \overrightarrow{\mathcal{E}}}{\partial t^{2}}=0
$$

Note que as equações $(80)$ é resultado do desacoplamento das equações $79 \mathrm{c}$ e $79 \mathrm{~d}$. Portanto, campos soluções das equações de ondas também são soluções dessas equações de Maxwell.

Podemos eliminar a derivada temporal da equação de onda supondo campos da forma

$$
\overrightarrow{\mathcal{E}}(x, y, z, t)=\vec{E}_{0}(x, y, z) e^{-i w t}
$$

ou

$$
\overrightarrow{\mathcal{B}}(x, y, z, t)=\vec{B}_{0}(x, y, z) e^{-i w t}
$$

Portanto, a equação de ondas origina uma equação de Helmholtz para os campos elétrico e magnético,

$$
\begin{aligned}
& \nabla^{2} \overrightarrow{\mathcal{E}}+\frac{\omega^{2}}{c^{2}} \overrightarrow{\mathcal{E}}=0 \\
& \nabla^{2} \overrightarrow{\mathcal{B}}+\frac{\omega^{2}}{c^{2}} \overrightarrow{\mathcal{B}}=0
\end{aligned}
$$

Repare que a equação de Helmholtz para os campos, como a equação de onda, é uma maneira de relacionar os campos de forma desacoplada. A equação (83), por exemplo, implica divergência nula de $\overrightarrow{\mathcal{E}}$ e a validade das equações (79c) e $79 \mathrm{~d}$, mas não garante (79b). Portanto, dada a solução da equação de Helmholtz para o campo elétrico, $\overrightarrow{\mathcal{B}}$ pode ser calculado pela Lei de Faraday, e satisfará a Lei de Ampère consequentemente, e sua divergência nula concede uma restrição entre as constantes da solução.

A solução geral da equação de Helmholtz para os campos são dadas por [17, 18]

$$
\begin{aligned}
\overrightarrow{\mathcal{E}}(x, y, z, t)= & A \cos \left(k_{x} x-\phi_{1}\right) \sin \left(k_{y} y\right) \sin \left(k_{z} z\right) \\
& \times e^{-i \omega t} \hat{i} \\
& +B \cos \left(k_{y} y-\phi_{2}\right) \sin \left(k_{x} x\right) \sin \left(k_{z} z\right) \\
& \times e^{-i \omega t} \hat{j} \\
& +C \cos \left(k_{z} z-\phi_{3}\right) \sin \left(k_{x} x\right) \sin \left(k_{y} y\right) \\
& \times e^{-i \omega t} \hat{k},
\end{aligned}
$$


$\mathrm{Ou}$

$$
\begin{aligned}
\overrightarrow{\mathcal{B}}(x, y, z, t)= & A \cos \left(k_{x} x-\phi_{1}\right) \sin \left(k_{y} y\right) \sin \left(k_{z} z\right) \\
& \times e^{-i \omega t} \hat{i} \\
& +B \cos \left(k_{y} y-\phi_{2}\right) \sin \left(k_{x} x\right) \sin \left(k_{z} z\right) \\
& \times e^{-i \omega t} \hat{j} \\
& +C \cos \left(k_{z} z-\phi_{3}\right) \sin \left(k_{x} x\right) \sin \left(k_{y} y\right) \\
& \times e^{-i \omega t} \hat{k},
\end{aligned}
$$

onde $A, B, C$ e $\phi$ são constantes arbitrárias.

Note que $\vec{k}$ real dá origem a senos e cossenos, cujo argumentos são uma função linear do espaço, proporcional a constante da direção correspondente, gerando ondas oscilantes; quando a constante for complexa, teremos funções seno e cosseno hiperbólicos, que dão origem a campos que decaem com o tempo. Além disso, evidentemente solução da equação de Helmholtz é da forma (81), ou (82), visto que essa solução foi a hipótese da qual a equação é advinda.

Para analisarmos a propagação da onda, vamos considerar uma caixa retangular com a superfície feita de um material perfeitamente condutor, preenchida por um dielétrico uniforme. Desse modo, a caixa retangular possuirá sua própria permissividade $(\mu)$ e permeabilidade $(\epsilon)$. Como consequência direta dessa configuração, devem desaparecer as componestes do campo nas paredes da caixa que são perpendiculares a direção de propagação da onda, o que nos fornece as condições de contorno, para parte espacial do campo,

$$
\left\{\begin{array}{l}
\vec{E}(x, y, z) \cdot \hat{i}=0 \rightarrow y=z=0 \text { ou } y=b, z=c \\
\vec{E}(x, y, z) \cdot \hat{j}=0 \rightarrow x=z=0 \text { ou } x=a, z=c \\
\vec{E}(x, y, z) \cdot \hat{k}=0 \rightarrow x=y=0 \text { ou } x=a, y=b
\end{array}\right.
$$

Com foco nas condições de contorno 85 e na solução de Helmholtz, pode-se observar que o campo eletrostático 78 pode ser usado como parte espacial de três ondas, em decaimento, superpostas. Desse modo, constata-se que as constantes de $\vec{k}$ para cada onda são dadas por

$$
\begin{aligned}
\vec{k}^{\{1\}} & =l_{1} \hat{i}+\frac{(2 n+1) \pi}{b} \hat{j}+\frac{(2 m+1) \pi}{c} \hat{k} \\
\vec{k}^{\{2\}} & =\frac{n \pi}{a} \hat{i}+\lambda_{2} \hat{j}+\frac{(2 m+1) \pi}{c} \hat{k} \\
\vec{k}^{\{3\}} & =\frac{(2 n+1) \pi}{a} \hat{i}+\frac{m \pi}{b} \hat{j}+k_{3} \hat{k}
\end{aligned}
$$

onde $l_{1}, \lambda_{2}$ e $k_{3}$ são definidos nas relações 66, 70 e (76), complexos, para possibilitar umas representação vetorial do campo elétrico. Portanto, senos e cossenos cujos argumentos são proporcionais a essas constantes, tornam-se hiperbólicos ao passo que adotamos $\bar{l}_{n, m}, \bar{\lambda}_{n, m}$ e $k_{n, m}$.

A constante $\phi$ é arbitrária e será definida como nula nos três campos. As constantes $A, B$ e $C$, no entanto, são obtidas imediatamente do campo (78),

$$
\begin{aligned}
& A_{n, m}^{\{1\}}=-\frac{16 V_{0} \bar{l}_{n, m}}{\sinh \left(\bar{l}_{n, m} a\right)(2 n+1)(2 m+1) \pi^{2}} \\
& A_{n, m}^{\{2\}}=-\frac{8 V_{0}(4 n+2)}{b \sinh \left(\bar{l}_{n, m} a\right)(2 n+1)(2 m+1) \pi} \\
& A_{n, m}^{\{3\}}=-\frac{8 V_{0}(4 m+2)}{\sinh \left(\bar{l}_{n, m} a\right)(2 n+1)(2 m+1) \pi} \\
& B_{n, m}^{\{1\}}=-\frac{8 V_{0} n(-1)^{n} \pi \sin a}{a \sinh \left(\bar{\lambda}_{n, m} b\right)(2 n+1)(2 m+1)} \\
& B_{n, m}^{\{2\}}=-\frac{8 V_{0} \bar{\lambda}_{n, m} n(-1)^{n} \sin a}{\sinh \left(\bar{\lambda}_{n, m} b\right)(2 n+1)(2 m+1)} \\
& B_{n, m}^{\{3\}}=-\frac{8 V_{0}(2 m+1) n(-1)^{n} \pi \sin a}{c \sinh \left(\bar{\lambda}_{n, m} b\right)(2 n+1)(2 m+1)} \\
& C_{n, m}^{\{1\}}=-\frac{8 V_{0} m \pi(2 n+1)\left[(-1)^{m} \cos b-1\right]}{a \sinh \left(k_{n, m} c\right)\left(b^{2}-\pi^{2} m^{2}\right)(2 n+1)} \\
& C_{n, m}^{\{2\}}=-\frac{8 V_{0} \pi m^{2}\left[(-1)^{m} \cos b-1\right]}{b \sinh \left(k_{n, m} c\right)\left(b^{2}-\pi^{2} m^{2}\right)(2 n+1)} \\
& C_{n, m}^{\{3\}}=-\frac{8 V_{0} k_{n, m} m\left[(-1)^{m} \cos b-1\right]}{\sinh \left(k_{n, m} c\right)\left(b^{2}-\pi^{2} m^{2}\right)(2 n+1)}
\end{aligned}
$$

Isto posto, os campos elétricos e magnéticos podem ser escritos vetorialmente na forma

$$
\begin{aligned}
\overrightarrow{\mathcal{E}}(x, y, z, t)= & \sum_{n, m, i} A_{n, m}^{\{i\}} \cos \left(k_{x}^{\{i\}} x\right) \sin \left(k_{y}^{\{i\}} y\right) \sin \left(k_{z}^{\{i\}} z\right) e^{-i \omega t} \hat{i} \\
& +\sum_{n, m, i} B_{n, m}^{\{i\}} \cos \left(k_{y}^{\{i\}} y\right) \sin \left(k_{x}^{\{i\}} x\right) \sin \left(k_{z}^{\{i\}} z\right) e^{-i \omega t} \hat{j} \\
& +\sum_{n, m, i} C_{n, m}^{\{i\}} \cos \left(k_{z}^{\{i\}} z\right) \sin \left(k_{x}^{\{i\}} x\right) \sin \left(k_{y}^{\{i\}} y\right) e^{-i \omega t} \hat{k} \\
\overrightarrow{\mathcal{B}}(x, y, z, t)= & \sum_{n, m, i} \mathcal{B}_{x}^{\{i\}}(x, y, z, t) \hat{i}+\sum_{n, m, i} \mathcal{B}_{y}^{\{i\}}(x, y, z, t) \hat{j}+\sum_{n, m, i} \mathcal{B}_{z}^{\{i\}}(x, y, z, t) \hat{k} .
\end{aligned}
$$


As componentes do campo magnético correspondente ao campo elétrico são determinadas através da Lei de Faraday,

$$
\begin{aligned}
& \mathcal{B}_{x}^{\{1\}}(x, y, z, t)=-\frac{i}{\omega} \sum_{n, m=0}^{\infty}\left[k_{z}^{\{1\}} B_{n, m}^{\{1\}}-k_{y}^{\{1\}} C_{n, m}^{\{1\}}\right] \sinh \left(\bar{l}_{n, m} x\right) \cos k_{y}^{\{1\}} y \cos k_{z}^{\{1\}} z e^{-i \omega t} \\
& \mathcal{B}_{y}^{\{1\}}(x, y, z, t)=-\frac{i}{\omega} \sum_{n, m=0}^{\infty}\left[C_{n, m}^{\{1\}} \bar{l}_{n, m}-k_{z}^{\{1\}} A_{n, m}^{\{1\}}\right] \cosh \left(\bar{l}_{n, m} x\right) \sin k_{y}^{\{1\}} y \cos k_{z}^{\{1\}} z e^{-i \omega t} \\
& \mathcal{B}_{z}^{\{1\}}(x, y, z, t)=-\frac{i}{\omega} \sum_{n, m=0}^{\infty}\left[k_{y}^{\{1\}} A_{n, m}^{\{1\}}-\bar{l}_{n, m} C_{n, m}^{\{1\}}\right] \cosh \left(\bar{l}_{n, m} x\right) \cos k_{y}^{\{1\}} y \sin k_{z}^{\{1\}} z e^{-i \omega t} \\
& \mathcal{B}_{x}^{\{2\}}(x, y, z, t)=-\frac{i}{\omega} \sum_{n, m=0}^{\infty}\left[k_{z}^{\{2\}} B_{n, m}^{\{2\}}-\bar{\lambda}_{n, m} C_{n, m}^{\{2\}}\right] \cosh \left(\bar{\lambda}_{n, m} y\right) \sin k_{x}^{\{2\}} x \cos k_{z}^{\{2\}} z e^{-i \omega t} \\
& \mathcal{B}_{y}^{\{2\}}(x, y, z, t)=-\frac{i}{\omega} \sum_{n, m=0}^{\infty}\left[k_{x}^{\{2\}} C_{n, m}^{\{2\}}-k_{z}^{\{2\}} A_{n, m}^{\{2\}}\right] \sinh \left(\bar{\lambda}_{n, m} y\right) \cos k_{x}^{\{2\}} x \cos k_{z}^{\{2\}} z e^{-i \omega t} \\
& \mathcal{B}_{z}^{\{2\}}(x, y, z, t)=-\frac{i}{\omega} \sum_{n, m=0}^{\infty}\left[\bar{\lambda}_{n, m} A_{n, m}^{\{2\}}-k_{x}^{\{2\}} B_{n, m}^{\{2\}}\right] \cosh \left(\bar{\lambda}_{n, m} y\right) \cos k_{x}^{\{2\}} x \sin k_{z}^{\{2\}} z e^{-i \omega t} \\
& \mathcal{B}_{x}^{\{3\}}(x, y, z, t)=-\frac{i}{\omega} \sum_{n, m=0}^{\infty}\left[k_{n, m} B_{n, m}^{\{3\}}-k_{y}^{\{3\}} C_{n, m}^{\{3\}}\right] \cosh \left(k_{n, m} z\right) \sin k_{x}^{\{3\}} x \cos k_{y}^{\{3\}} y e^{-i \omega t} \\
& \mathcal{B}_{z}^{\{3\}}(x, y, z, t)=-\frac{i}{\omega} \sum_{n, m=0}^{\infty}\left[k_{y}^{\{3\}} A_{n, m}^{\{3\}}-k_{x}^{\{3\}} B_{n, m}^{\{3\}}\right] \sinh \left(k_{n, m} z\right) \cos k_{x}^{\{3\}} x \cos k_{y}^{\{3\}} y e^{-i \omega t} \\
& \mathcal{B}_{y}^{\{3\}}(x, y, z, t)=-\frac{i}{\omega} \sum_{n, m=0}^{\infty}\left[k_{x}^{\{3\}} C_{n, m}^{\{3\}}-k_{n, m} A_{n, m}^{\{3\}}\right] \cosh \left(k_{n, m} z\right) \cos k_{x}^{\{3\}} x \sin k_{y}^{\{3\}} y e^{-i \omega t}
\end{aligned}
$$

Outrossim, para que os campos elétricos estejam de acordo com a equação de onda e possuam divergência nula, as constantes relacionam-se de modo que

$$
\begin{gathered}
\sum_{i=0}^{3}\left(k_{x}^{\{i\}} A_{n, m}^{\{i\}}+k_{y}^{\{i\}} B_{n, m}^{\{i\}}+k_{z}^{\{i\}} C_{n, m}^{\{i\}}\right)=0 \\
\sum_{i=0}^{3}\left|\vec{k}^{\{i\}}\right|^{2}=\frac{3 \omega^{2}}{c^{2}}
\end{gathered}
$$

Note que as constante $l_{1}, \lambda_{2}, \quad$ e $k_{3}$ em 86 são imaginárias puras. Quando substituídas na expressão do campo elétrico (96), passam a ser os reais $\bar{l}_{n, m}, \bar{\lambda}_{n, m}$, e $k_{n, m}$ ao posso que surgem senos e cossenos hiperbólicos, os quais estão presentes nas expressões para as componentes dos campos magnéticos 98 106. Observe, também, que a parte real do campo magnético é proporcional ao sin $(\omega t)$ de forma que se anula em $t=0$ e o campo elétrico 96 assumi a forma do campo eletrostático (78), como deveria ser.

As soluções (96) e (97) são amplamente usadas em cavidades ressoantes e guias de ondas [3, 4, 17, 10, 17, 18, de forma similar a propagação de campos no interior de um forno mrico-ondas [19].

\section{Conclusão}

Nesse artigo estudamos em detalhes o problema da solução da equação de Laplace para uma placa finita bidimensional e uma caixa retangular tridimensional. Para isso, usamos o método de separação de variáveis e coeficientes de Fourier, para assim determinar soluções que satisfazem condições iniciais específicas. No caso da caixa retangular tridimensional, as condições iniciasi para o potencial eletrostático pode levar a resulução individual da equação de Laplace, em cada caso, e ao final utilizar o teorema da superposição de EDPs para essas soluções. Esse método é sempre sugerido pelos livros textos mas nunca explicitado, o que fazemos claramente aqui.

Fazemos também uma aplicação em que consideramos a equação de Helmholtz para os campos elétrico e magnético. A solução dessa equação é a soma de uma solução particular, solução da equação inomogênea, mais a solução homogênea, a qual é solução da equação de Laplace. Sendo assim, a solução de Laplace continua sendo de fundamental importância mesmo em fenômenos eletrodinâmicos, como propagação de ondas eletromagnéticas em guias de ondas, cavidades ressonantes e forno micro-ondas. Nesse caso, obtemos a 
solução dos campos elétrico e magnético dinâmicos, e verificamos que o campo elétrico é idênticos ao campo eletrostático para quando o tempoe é $t=0$, ou seja, o campo advindo do potencial que é solução da equação de Laplace.

\section{Agradecimentos}

Os autores agradecem aos professores Davi C. Rodrigues e Danilo T. Alves por auxílios na plotagem dos campos e ao CNPq pelas bolsas PIBIC e financiamento parcial do trabalho. Os autores agradecem a um árbitro anônimo por sugestões que trouxeram melhorias ao trabalho.

\section{Referências}

[1] D.W. Hahn e M.N. Ozisik, Heat Conduction (John Wiley, New Jersey 2012).

[2] W. Greiner e J. Reinhardt, Field Quantization (Springer-Verlag, Berlin 1996).

[3] D.J. Griffiths Mathematical Methods for Physicists (Elsevier, New Jersy, 1999), p. 576, $3^{\mathrm{a}}$ ed.

[4] J.D. Jackson, Classical Electrodynamics (Wiley, New Jersy, 1999), p. 808, 3 $3^{\mathrm{a}}$ ed.

[5] J.R. Reitz, F.J. Milford e R.W. Christy, Foundations of Electromagnetic Theory (Addison Wesley, Boston, 1992), p. 387, $4^{\text {a }}$ ed.

[6] K.D. Machado, Teoria do electromagnetismo (Universidade Estadual de Ponta Grossa, Ponta Grossa, 2002), v. 1, p. $929,1^{\mathrm{a}}$ ed.

[7] W. Greiner, Classical Electrodynamics (Springer, New York, 1998), p. 555.

[8] A.F.T. Carvalho, Problema de Dirichlet: métodos de resolução. Dissertação de Mestrado, Universidade Estadual de Campinas, Campinas (1994).

[9] D.T. Alves, E.R. Granhen, J.P.S. Alves e W.A. Lima, arXiv:2008.08167v2 (2020).

[10] K.D. Machado, Teoria do electromagnetismo (Universidade Estadual de Ponta Grossa, Ponta Grossa, 2006), v. 3 , p. 1100, $1^{\text {a }}$ ed.

[11] F.J. Santos, Introdução às Séries de Fourier, disponível em: http://www.matematica.pucminas.br/profs/web_f abiano/calculo4/sf.pdf, acessado em 04/10/2020.

[12] G.B. Arfken e H.J. Weber, Mathematical Methods for Physicists (Elsevier, Amsterdã, 2005), p. 1182, $6^{\mathrm{a}}$ ed.

[13] R.J. Biezuner, Notas de Aula Equações Diferenciais Parciais I/II, disponível em: https://paca.ime.usp.b r/pluginfile.php/42464/course/section/10220/Biezune r\%2CR.J.\%20\%20Notas\%20de\%20aula\%20de\%20quaco es\%20diferenciais\%20parciais\%20I\%20e\%20II.2010.pdf acessado em 08/07/2021.

[14] M. Furtado, Notas de EDP2 (versão 1.2), disponível em: https://www.mat.unb.br/ furtado/homepage/notasedp2.pdf, acessado em: 08/07/2021.

[15] R. Robert, Rev. Bras. Ensino Fís. 22, 2 (2000).
[16] R. Robert, S.L.M. Berleze e V.S. Filho, Rev. Bras. Ensino Fís. 27, 4 (2005).

[17] University of Liverpool, Waves on boundaries, disponível em: http://pcwww.liv.ac.uk/ awolski/main_teaching_L iverpool_PHYS370.htm, acesso em 03/10/2020.

[18] E. Jensen, RF Basics and TM Cavities, disponível em: https://indico.cern.ch/event/626653/attachments/1524 529/2383307/01_-_RF_Basics_and_TM_Cavities_v2.pdf acesso em 03/10/2020.

[19] Ressoadores eletromagnéticos, disponível em: https://ed isciplinas.usp.br/pluginfile.php/5519146/mod_resource/ content/4/7-Ressoadores\%20Elestromagneticos\%20-\% 20PSI3483\%20-2020.pdf , acesso em 13/01/2021. 